\title{
Description of a new horned toad of Megophrys Kuhl \& Van Hasselt, 1822 (Anura, Megophryidae) from southwest China
}

\author{
Haijun $\mathrm{Su}^{{ }^{*}}$, Shengchao $\mathrm{Shi}^{2 *}$, Yanqing $\mathrm{Wu}^{3}$, Guangrong $\mathrm{Li}^{4}$, \\ Xiaogang $\mathrm{Yao}^{4}$, Bin Wang ${ }^{2}$, Shize $\mathrm{Li}^{2}$ \\ I College of Forestry, Guizhou University, Guiyang 550002, China 2 Chengdu Institute of Biology, Chinese \\ Academy of Sciences, Chengdu 610041, China 3 Nanjing Institute of Environmental Sciences, Ministry of \\ Ecology and Environment of China, Nanjing 210042, China 4 Kuankuoshui National Nature Reserve Ad- \\ ministration, Suiyang 563300, China
}

Corresponding author: Shize Li (976722439@qq.com); Bin Wang (wangbin@cib.ac.cn)

Academic editor: Anthony Herrel | Received 1 July 2020 | Accepted 24 August 2020 | Published 7 October 2020

http://zoobank.org/42BA600C-5E98-4408-8C4E-2CC8339D582A

Citation: Su H, Shi S, Wu Y, Li G, Yao X, Wang B, Li S (2020) Description of a new horned toad of Megophrys Kuhl \& Van Hasselt, 1822 (Anura, Megophryidae) from southwest China. ZooKeys 974: 131-159. https://doi.org/10.3897/ zookeys. 974.56070

\begin{abstract}
A new species of the genus Megophrys is described from Guizhou Province, China. Molecular phylogenetic analyses supported the new species as an independent clade nested into the Megophrys. The new species could be distinguished from its congeners by a combination of the following characters: body size moderate (SVL 49.3-58.2 $\mathrm{mm}$ in males); vomerine ridges present distinctly, vomerine teeth present; tongue feebly notched behind; tympanum distinctly visible, oval; two metacarpal tubercles in hand; toes with one-third webbing and wide lateral fringes; heels overlapped when thighs are positioned at right angles to the body; tibiotarsal articulation reaching the level between tympanum and eye when leg stretched forward; an internal single subgular vocal sac present in male; in breeding male, the nuptial pads with large and sparse black nuptial spines present on the dorsal bases of the first two fingers.
\end{abstract}

\section{Keywords}

China, molecular phylogenetic analysis, morphology, new species, taxonomy

* Contributed equally as the first author.

Copyright Haijun Su et al. This is an open access article distributed under the terms of the Creative Commons Attribution License (CC BY 4.0), which permits unrestricted use, distribution, and reproduction in any medium, provided the original author and source are credited. 


\section{Introduction}

The toad genus Megophrys Kuhl \& Van Hasselt, 1822 (Anura; Megophryidae) is widely distributed in eastern and central China, throughout southeastern Asia, and extending to the islands of the Sunda Shelf and the Philippines (Frost 2020). The taxonomic assignments of the taxa in this group have been debated for a long time (e.g., Tian and Hu 1983; Dubois 1987; Lathrop 1997; Rao and Yang 1997; Jiang et al. 2003; Delorme et al. 2006; Fei et al. 2009; Chen et al. 2016; Fei and Ye 2016; Deuti et al. 2017; Mahony et al. 2017; Frost 2020). Regardless, molecular phylogenetic studies indicate the group as a monophyletic group (Chen et al. 2017; Mahony et al. 2017; Li et al. 2018b; Liu et al. 2018, 2020; Wang et al. 2020), and thus some studies regarded it as a large genus, Megophrys sensu lato (Mahony et al. 2017; Li et al. 2018b; Liu et al. 2018, 2020; Wang et al. 2020; Frost 2020) while other studies divided the taxon into different genera and subgenera (Chen et al. 2017; Fei and Ye 2016; Liu et al. 2018).

The genus Megophrys currently contains 106 species, of which 50 species have been described in the last decade (Frost 2020). Many cryptic species were indicated in the genus by molecular phylogenetic frameworks (Chen et al. 2017; Liu et al. 2018). In recent years, four species were described from Guizhou Province, China: Megophrys liboensis Zhang, Li, Xiao, Li ,Pan, Wang, Zhang \& Zhou, 2017, Megophrys leishanensis Li, Xu, Liu, Jiang, Wei \& Wang, 2018, Megophrys jiangi Liu, Li, Wei, Xu, Cheng, Wang \& Wu, 2020, and Megophrys chishuiensis Xu, Li, Liu, Wei, \& Wang, 2020. However, many areas have not been well investigated in this province, and it is expected that there are still cryptic species of the toads in the region.

During field surveys in the Huanglian Nature Reserve, Tongzi County, and Kuankuoshui National Nature Reserve, Suiyang County in Guizhou Province, China, we collected a number of Megophrys specimens. Molecular phylogenetic analyses, morphological comparisons, and bioacoustics data support it as an undescribed species.

\section{Materials and methods}

\section{Sampling}

A total of nine molecular samples were collected in this study: five adult males of the undescribed species from two localities of Guizhou Province, China, two M. sangzhiensis and two M. spinata (Table 1; Fig. 1). In the field, the toads were euthanized using isoflurane, and the specimens were fixed in $75 \%$ ethanol. Tissue samples were taken and preserved separately in $99 \%$ ethanol prior to fixation. The specimens were deposited in Chengdu Institute of Biology, Chinese Academy of Sciences (CIB, CAS). 


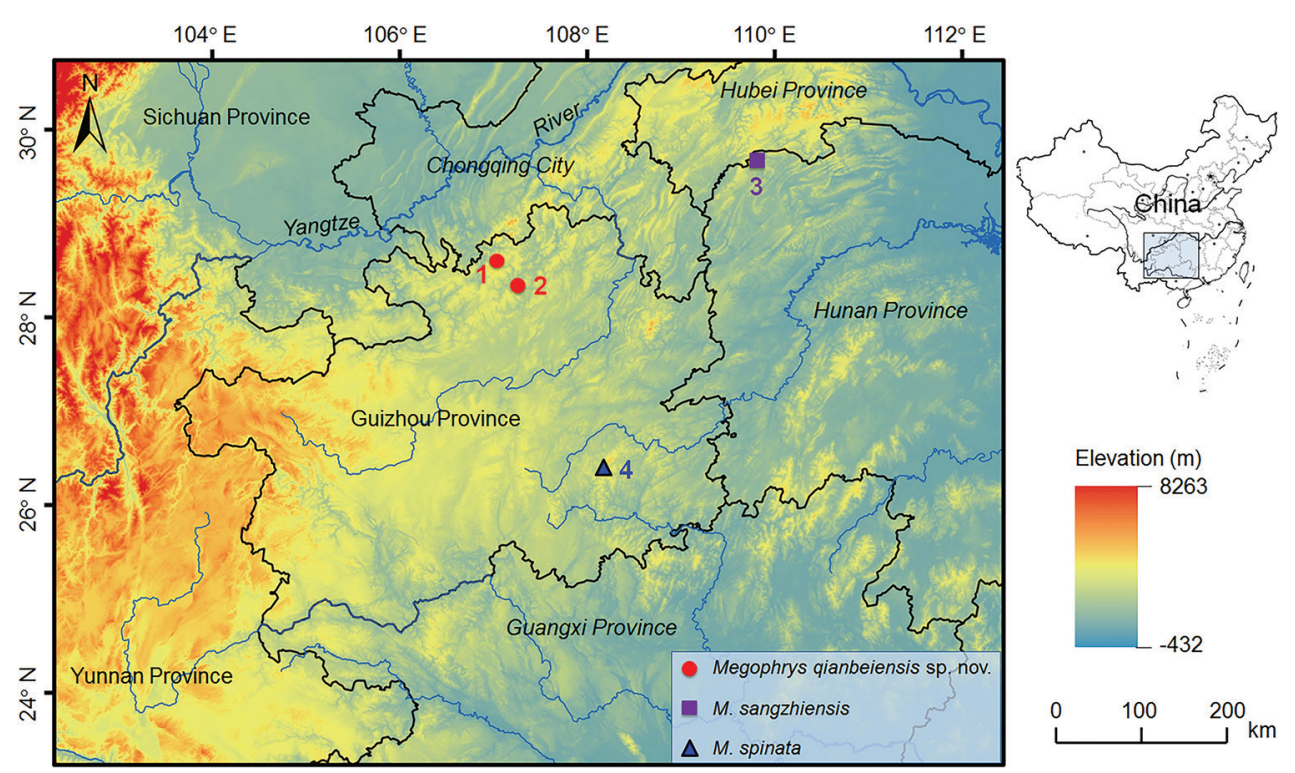

Figure I. Sampling localities of Megophrys qianbeiensis sp. nov., M. sangzhiensis and M. spinata in China. 1. Huanglian Nature Reserve, Tongzi County, Guizhou Province; 2. Kuankuoshui National Nature Reserve, Suiyang County, Guizhou Province; 3. Badagong Mountain, Hunan Province, China; 4. Leigong Mountain, Guizhou Province.

\section{Molecular data and phylogenetic analyses}

Total DNA was extracted using a standard phenol-chloroform extraction protocol (Sambrook et al. 1989). Two fragments of the mitochondrial $16 \mathrm{~S}$ rRNA (16S) and cytochromeoxidase subunit I (COI) genes were amplified. For 16S, the primers P7 (5'-CGCCTGTTTACCAAAAACAT-3') and P8 (5'-CCGGTCTGAACTCAGATCACGT-3') were used following Simon et al. (1994), and for COI, Chmf4 (5'-TYTCWACWAAYCAYAAAGAYATCGG-3') and Chmr4 (5'-ACYTCRGGRTGRCCRAARAATCA-3') were used following Che et al. (2012). Gene fragments were amplified under the following conditions: an initial denaturing step at $95^{\circ} \mathrm{C}$ for $4 \mathrm{~min} ; 36$ cycles of denaturing at $95{ }^{\circ} \mathrm{C}$ for $30 \mathrm{~s}$, annealing at $52{ }^{\circ} \mathrm{C}$ (for $16 \mathrm{~S}$ ) $/ 47^{\circ} \mathrm{C}$ (for COI) for $40 \mathrm{~s}$ and extending at $72{ }^{\circ} \mathrm{C}$ for $70 \mathrm{~s}$. Sequencing was conducted using an ABI3730 automated DNA sequencer in Shanghai DNA BioTechnologies Co., Ltd. (Shanghai, China). New sequences were deposited in GenBank (for accession numbers see Table 1).

For molecular analyses, the available sequence data for congeners of Megophrys were downloaded from GenBank (Table 1), primarily from previous studies (Chen et al. 2017; Liu et al. 2018). For phylogenetic analyses, corresponding sequences of one Leptobrachella oshanensis (Liu, 1950) and one Leptobrachium boringii (Liu, 1945) were also downloaded (Table 1), and used as outgroups according to Mahony et al. (2017). Sequences were assembled and aligned using the Clustalw module in BioEdit v.7.0.9.0 
Table I. Information for samples used in molecular phylogenetic analyses in this study.

\begin{tabular}{|c|c|c|c|c|c|}
\hline \multirow[t]{2}{*}{ ID } & \multirow[t]{2}{*}{ Species } & \multirow[t]{2}{*}{ Voucher number } & \multirow[t]{2}{*}{ Locality } & \multicolumn{2}{|c|}{ GenBank accession number } \\
\hline & & & & $16 \mathrm{~S}$ & COI \\
\hline 1 & Megophrys qianbeiensis sp. nov. & CIBTZ20190608015 & Huanglian Nature Reserve, Guizhou, China & MT651553 & MT654520 \\
\hline 2 & Megophrys qianbeiensis sp. nov. & CIBTZ20190608017 & Huanglian Nature Reserve, Guizhou, China & MT651554 & MT654521 \\
\hline 3 & Megophrys qianbeiensis sp. nov. & CIBTZ20160715003 & Huanglian Nature Reserve, Guizhou, China & MT651555 & MT654522 \\
\hline 4 & Megophrys qianbeiensis sp. nov. & CIBKKS20180722002 & Huanglian Nature Reserve, Guizhou, China & MT651556 & MT654523 \\
\hline 5 & Megophrys qianbeiensis sp. nov. & CIBKKS20180722001 & Kuankuoshui Nature Reserve, Guizhou, China & MT651557 & MT654524 \\
\hline 6 & Megophrys sangzhiensis & CIBSZ2012062005 & Badagongshan Nature Reserve, Hunan, China & MT651558 & MT654525 \\
\hline 7 & Megophrys sangzhiensis & CIBSZ2012062008 & Badagongshan Nature Reserve, Hunan, China & MT651559 & MT654526 \\
\hline 8 & Megophrys sangzhiensis & SYSa004307 & Zhangjiajie, Hunan, China & MH406798 & MH406260 \\
\hline 9 & Megophrys spinata & CIBLS20190801001 & Leigong Shan, Guizhou, China & MT651551 & MT654518 \\
\hline 10 & Megophrys spinata & CIBLS20190801002 & Leigong Shan, Guizhou, China & MT651552 & MT654519 \\
\hline 11 & Megophrys spinata & SYSa002227 & Leigong Shan, Guizhou, China & MH406676 & MH406116 \\
\hline 12 & Megophrys binlingensis & KIZ025807 & Wawu Shan, Sichuan, China & KX811852 & KX812115 \\
\hline 13 & Megophrys binlingensis & SYSa005313 & Wawu Shan, Sichuan, China & MH406892 & MH406354 \\
\hline 14 & Megophrys binlingensis & SYSa005314 & Wawu Shan, Sichuan, China & MH406893 & MH406355 \\
\hline 15 & Megophrys binchuanensis & KIZ019441 & Jizu Shan, Yunnan, China & KX811849 & KX812112 \\
\hline 16 & Megophrys palpebralespinosa & KIZ011603 & Pu Hu Nature Reserve, Thanh Hoa, Vietnam & KX811888 & KX812137 \\
\hline 17 & Megophrys omeimontis & KIZ025765 & Emei Shan, Sichuan, China & KX811884 & KX812136 \\
\hline 18 & Megophrys angka & KIZ040591 & Kiew Mae Pan nature trail, Chiang Mai, Thailand & MN508052 & - \\
\hline 19 & Megophrys wuliangshanensis & KIZ046812 & Huangcaoling, Yunnan, China & KX811881 & KX812129 \\
\hline 20 & Megophrys daweimontis & KIZ048997 & Dawei Shan, Yunnan, China & KX811867 & KX812125 \\
\hline 21 & Megophrys jingdongensis & KIZ-LC0805067 & $\begin{array}{l}\text { Huanglianshan National Nature Reserve, Yunnan, } \\
\text { China }\end{array}$ & KX811872 & KX812131 \\
\hline 22 & Megophrys fansipanensis & VNMN 2018.01 & Lao Cai, Sa Pa, Vietnam & MH514886 & - \\
\hline 23 & Megophrys hoanglienensis & VNMN 2018.02 & Lao Cai, Sa Pa, Vietnam & MH514889 & - \\
\hline 24 & Megophrys minor & KIZ01939 & Qingcheng Shan, Sichuan, China & KX811896 & KX812145 \\
\hline 25 & Megophrys jiangi & CIBKKS20180722006 & Kuankuosui Nature Reserve, Guizhou, China & MN107743 & MN107748 \\
\hline 26 & Megophrys chishuiensis & CIBCS20190518031 & Chishui Nature Reserve, Guizhou, China & MN954707 & MN928958 \\
\hline 27 & Megophrys dongguanensis & SYS a001972 & Yinping Shan, Guangdong, China & MK524098 & MK524129 \\
\hline 28 & Megophrys nankunensis & SYS a004498 & Nankun Shan, Guangdong, China & MK524108 & MK524139 \\
\hline 29 & Megophrys cheni & SYS a001427 & Jinggang Shan, Jiangxi, China & KJ560391 & - \\
\hline 30 & Megophrys obesa & SYS a002272 & Heishiding Nature Reserve, Guangdong, China & KJ579122 & - \\
\hline 31 & Megophrys ombrophila & KRM18 & Wuyishan, Fujian, China & KX856404 & - \\
\hline 32 & Megophrys wugongensis & SYS a002610 & Wugongshan Scenic Area, Jiangxi, China & MK524114 & MK524145 \\
\hline 33 & Megophrys lini & SYS a002370 & Suichuan, Jiangxi, China & KJ560412 & - \\
\hline 34 & Megophrys xiangnanensis & SYS a002874 & Yangming Shan, Hunan, China & MH406713 & MH406165 \\
\hline 35 & Megophrys nanlingensis & SYS a001959 & Nanling Nature Reserve, Guangdong, China & MK524111 & MK524142 \\
\hline 36 & Megophrys kuatunensis & SYS a001579 & Wuyi Shan, Fujian, China & KJ560376 & - \\
\hline 37 & Megophrys jinggangensis & KIZ07132 & Chashan Forest Farm, Jiangxi, China & KX811840 & KX812108 \\
\hline 38 & Megophrys lishuiensis & WYF00169 & Lishui, Zhejiang, China & KY021418 & - \\
\hline 39 & Megophrys xianjuensis & CIBXJ190505 & Xianju, Zhejiang, China & MN563753 & MN563769 \\
\hline 40 & Megophrys wushanensis & KIZ045469 & Guangwu Shan, Sichuan, China & KX811838 & KX812094 \\
\hline 41 & Megophrys baolongensis & KIZ019216 & Baolong, Chongqing, China & KX811813 & KX812093 \\
\hline 42 & Megophrys leishanensis & CIBLS20171101001 & Leigong Shan, Guizhou, China & MK005310 & MK005306 \\
\hline 43 & Megophrys yangmingensis & SYS a002877 & Yangming Shan, Hunan, China & MH406716 & MH406168 \\
\hline 44 & Megophrys shimentaina & SYS a002077 & Shimentai Nature Reserve, Guangdong, China & MH406655 & MH406092 \\
\hline 45 & Megophrys jiulianensis & SYS a002107 & Jiulian Shan, Jiangxi, China & MK524099 & MK524130 \\
\hline 46 & Megophrys shunhuangensis & HNNU16SH02 & Shunhuang Mountains, Hunan, China & MK836037 & - \\
\hline 47 & Megophrys mirabilis & SYS a002192 & Huaping Nature Reserve, Guangxi, China & MH406669 & MH406109 \\
\hline 48 & Megophrys tuberogranulata & Tissue ID: YPX10987 & Badagongshan Nature Reserve, Hunan, China & KX811823 & KX812095 \\
\hline 49 & Megophrys huangshanensis & KIZ022004 & Huang Shan, Anhui, China & KX811821 & KX812107 \\
\hline 50 & Megophrys boettgeri & Tissue ID: YPXJK033 & Wuyi Shan, Fujian, China & KX811814 & KX812104 \\
\hline 51 & Megophrys liboensis & GNUG:20160408003 & Libo, Guizhou, China & MF285262 & - \\
\hline 52 & Megophrys mufumontana & SYS a006391 & Mufu Shan, Hunan, China & MK524105 & MK524136 \\
\hline 53 & Megophrys brachykolos & ROM 16634 & Hong Kong, China & KX811897 & KX812150 \\
\hline 54 & Megophrys acuta & SYS a001957 & Heishiding Nature Reserve, Guangdong, China & KJ579118 & - \\
\hline 55 & Megophrys gerti & ITBCZ 1108 & Nui Chua National Park, Ninh Thuan, Vietnam & KX811917 & KX812161 \\
\hline 56 & Megophrys elfina & ZMMU ABV-00454 & Bidoup Mountain, Lam Dong, Vietnam & KY425379 & - \\
\hline
\end{tabular}




\begin{tabular}{|c|c|c|c|c|c|}
\hline \multirow[t]{2}{*}{ ID } & \multirow[t]{2}{*}{ Species } & \multirow[t]{2}{*}{ Voucher number } & \multirow[t]{2}{*}{ Locality } & \multicolumn{2}{|c|}{ GenBank accession number } \\
\hline & & & & $16 S$ & COI \\
\hline 57 & Megophrys synoria & FMNH 262778 & O'Reang, Mondolkiri, Cambodia & KY022198 & - \\
\hline 58 & Megophrys hansi & KIZ010360 & $\begin{array}{c}\text { Phong Dien Nature Reserve, Thua Thien Hue, } \\
\text { Vietnam }\end{array}$ & KX811913 & KX812155 \\
\hline 59 & Megophrys microstoma & KIZ048799 & Xiaoqiaogou Nature Reserve, Yunnan, China & KX811914 & KX812156 \\
\hline 60 & Megophrys pachyproctus & KIZ010978 & Beibeng, Xizang, China & KX811908 & KX812153 \\
\hline 61 & Megophrys baluensis & ZMH A13125 & $\begin{array}{c}\text { Gunung Kinabalu National Park, Kogopan Trail, } \\
\text { Malaysia }\end{array}$ & KJ831310 & - \\
\hline 62 & Megophrys stejnegeri & KU 314303 & Pasonanca Natural Park, Zamboanga, Philippines & KX811922 & KX812052 \\
\hline 63 & Megophrys ligayae & ZMMU NAP-05015 & Palawan, Philippines & KX811919 & KX812051 \\
\hline 64 & Megophrys kobayashii & UNIMAS 8148 & Gunung Kinabalu National Park, Sabah, Malaysia & KJ831313 & - \\
\hline 65 & Megophrys nasuta & KIZ019419 & Malaysia & KX811921 & KX812054 \\
\hline 66 & Megophrys edwardinae & FMNH 273694 & Bintulu, Sarawak, Malaysia & KX811918 & KX812050 \\
\hline 67 & Megophrys aceras & KIZ025467 & $\begin{array}{c}\text { Khao Nan National Park, Nakhon Si Thammarat, } \\
\text { Thailand }\end{array}$ & KX811925 & KX812159 \\
\hline 68 & Megophrys dringi & UNIMAS 8943 & Gunung Mulu National Park, Sarawak, Malaysia & KJ831317 & - \\
\hline 69 & Megophrys maosonensis & KIZ016045 & Xiaoqiaogou Nature Reserve, Yunnan, China & KX811780 & KX812080 \\
\hline 70 & Megophrys mangshanensis & KIZ021786 & Nanling National Forest Park, Guangdong, China & KX811790 & KX812079 \\
\hline 71 & Megophrys flavipunctata & SDBDU2009.297 & East Khasi Hills dist., Meghalaya & KY022307 & MH647536 \\
\hline 72 & Megophrys glandulosa & KIZ048439 & Husa, Yunnan, China & KX811762 & KX812075 \\
\hline 73 & Megophrys medogensis & KIZ06621 & Beibeng, Xizang, China & KX811767 & KX812082 \\
\hline 74 & Megophrys periosa & BNHS 6061 & West Kameng dist., Arunachal Pradesh, IN & KY022309 & MH647528 \\
\hline 75 & Megophrys himalayana & SDBDU2009.75 & East Siang dist., Arunachal Pradesh, IN & KY022311 & - \\
\hline 76 & Megophrys sanu & K5198/ZSI11393 & - & KX894679 & - \\
\hline 77 & Megophrys zhangi & KIZ014278 & Zhangmu, Xizang, China & KX811765 & KX812084 \\
\hline 78 & Megophrys katabhako & ZSIA11799 & - & KX894669 & - \\
\hline 79 & Megophrys major & SYSa002961 & Zhushihe, Yunnan, China & MH406728 & MH406180 \\
\hline 80 & Megophrys oreocrypta & BNHS 6046 & West Garo Hills dist., Meghalaya & KY022306 & - \\
\hline 81 & Megophrys auralensis & NCSM 79599 & Aural, Kampong Speu, Cambodia & KX811807 & - \\
\hline 82 & Megophrys parva & SYSa003042 & Zhushihe, Yunnan, China & MH406737 & MH406189 \\
\hline 83 & Megophrys nankiangensis & CIB ZYC517 & Nanjiang, Sichuan, China & KX811900 & - \\
\hline 84 & Megophrys wawuensis & KIZ025799 & Wawu Shan, Sichuan, China & KX811902 & KX812062 \\
\hline 85 & Megophrys gigantica & SYSa003933 & Wuliang shan, Yunnan, China & MH406775 & MH406235 \\
\hline 86 & Megophrys shapingensis & KIZ014512 & Liziping Nature Reserve, Sichuan, China & KX811904 & KX812060 \\
\hline 87 & Megophrys montana & LSUMZ 81916 & Sukabumi, Java, Indonesia & KX811927 & KX812163 \\
\hline 88 & Megophrys lancip & MZB:Amp:22233 & - & KY679891 & - \\
\hline 89 & Megophrys feae & KIZ046706 & Huangcaoling, Yunnan, China & KX811810 & KX812056 \\
\hline 90 & Megophrys chuannanensis & CIB20050081 & Hejiang, Sichuan, China & KM504261 & - \\
\hline 91 & Megophrys carinense & Tissue ID: YPX20455 & Dayao Shan, Guangxi, China & KX811811 & KX812057 \\
\hline 92 & Megophrys popei & SYS a000589 & Naling Nature Reserve, Guangdong, China & KM504251 & - \\
\hline 93 & Megophrys intermedia & ZFMK 87596 & U Bo, Phong Nha-Ke Bang NP, Vietnam & HQ588950 & - \\
\hline 94 & Leptobrachium boringii & Tissue ID: YPX37539 & Emei Shan, Sichuan, China & KX811930 & KX812164 \\
\hline 95 & Leptobrachella oshanensis & KIZ025778 & Emei Shan, Sichuan, China & KX811928 & KX812166 \\
\hline
\end{tabular}

(Hall 1999) with default settings. Alignments were checked by eye and revised manually if necessary. For phylogenetic analyses of mitochondrial DNA, the dataset concatenated with $16 \mathrm{~S}$ and COI gene sequences. To avoid under- or over-parameterization (Lemmon and Moriarty 2004; McGuire et al. 2007), the best partition scheme and the best evolutionary model for each partition were chosen for the phylogenetic analyses using PARTITIONFINDER v. 1.1.1 (Robert et al. 2012). In this analysis, $16 S$ gene and each codon position of COI gene were defined, and Bayesian Inference Criteria was used. As a result, the analysis suggested that the best partition scheme is $16 \mathrm{~S}$ gene/each codon position of COI gene, and selected GTR + G + I model as the best model for each partition. Phylogenetic analyses were conducted using maximum likelihood (ML) 
and Bayesian Inference (BI) methods, implemented in PhyML v. 3.0 (Guindon et al. 2010) and MrBayes v. 3.12 (Ronquist and Huelsenbeck 2003), respectively. For the ML tree, branch supports were drawn from 10,000 nonparametric bootstrap replicates. In BI, two runs each with four Markov chains were simultaneously run for 50 million generations with sampling every 1,000 generations. The first $25 \%$ trees were removed as the "burn-in" stage followed by calculations of Bayesian posterior probabilities and the $50 \%$ majority-rule consensus of the post burn-in trees sampled at stationarity.

\section{Morphological comparisons}

In total, 16 specimens including six males of the undescribed species, five males of $M$. sangzhiensis, and five males of M. spinata were measured (for voucher information see Table 2). The terminology and methods followed Fei et al. (2009). Measurements were taken with a dial caliper to $0.1 \mathrm{~mm}$. Twenty-one morphometric characters of adult specimens were measured:

ED eye diameter (distance from the anterior corner to the posterior corner of the eye);

FL foot length (distance from tarsus to the tip of fourth toe);

HDL head length (distance from the tip of the snout to the articulation of jaw);

HDW maximum head width (greatest width between the left and right articulations of jaw);

HLL hindlimb length (maximum length from the vent to the distal tip of the Toe IV);

IAE distance between posterior corner of eyes;

IFE distance between anterior corner of eyes;

IND internasal distance (minimum distance between the inner margins of the external nares);

IOD interorbital distance (minimum distance between the inner edges of the upper eyelids);

LAL length of lower arm and hand (distance from the elbow to the distal end of the Finger IV);

LW lower arm width (maximum width of the lower arm);

NED nasal to eye distance (distance between the nasal and the anterior corner of the eye);

NSD nasal to snout distance (distance between the nasal the posterior edge of the vent);

SVL snout-vent length (distance from the tip of the snout to the posterior edge of the vent);

SL snout length (distance from the tip of the snout to the anterior corner of the eye);

TFL length of foot and tarsus (distance from the tibiotarsal articulation to the distal end of the Toe IV);

THL thigh length (distance from vent to knee);

TL tibia length (distance from knee to tarsus);

TW maximal tibia width;

TYD maximal tympanum diameter;

UEW upper eyelid width (greatest width of the upper eyelid margins measured perpendicular to the anterior-posterior axis). 


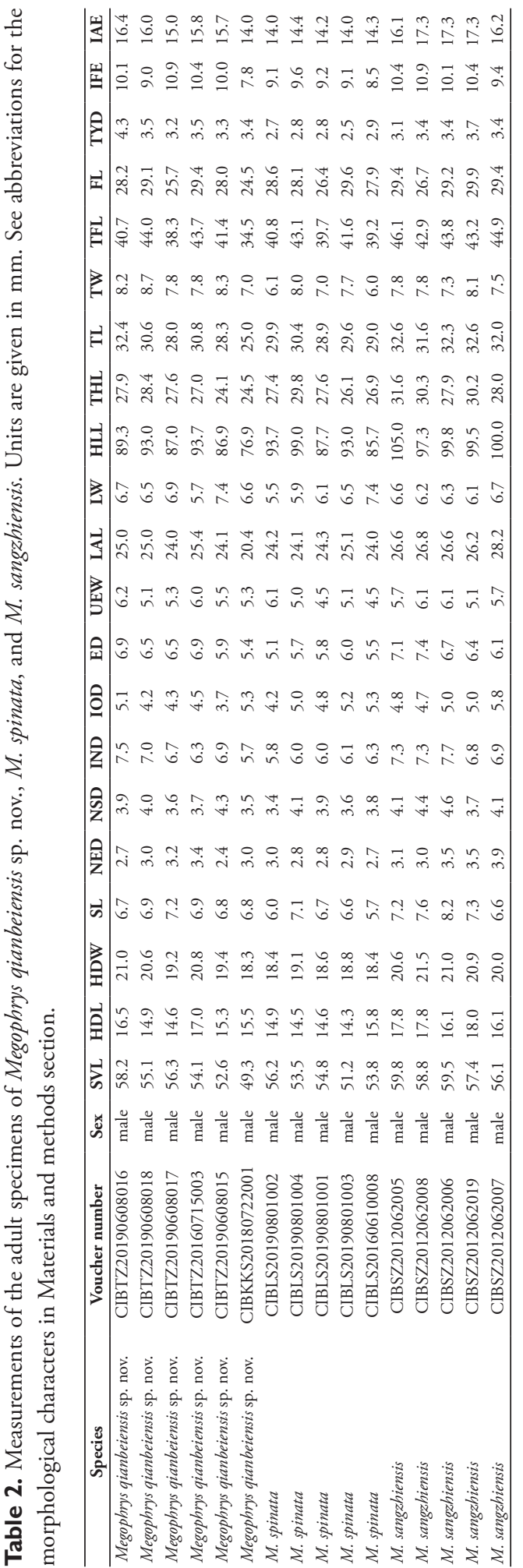


In order to reduce the impact of allometry, the correct value from the ratio of each character to SVL was calculated, and then was log-transformed for subsequent morphometric analyses. One-way analysis of variance (ANOVA) was used to test the significance of differences on morphometric characters between different species. The significance level was set at 0.05 . To show the spatial distribution of different species on the morphometric characters, principal component analyses (PCA) were performed. These analyses were carried out in the R (R Development Core Team 2008). The new species was also compared with all other Megophrys species on morphology. Comparative data were obtained from related species as described in literature (Table 3).

Table 3. References for morphological characters for congeners of the genus Megophrys.

\begin{tabular}{|c|c|}
\hline Species & Literature \\
\hline M. aceras Boulenger, 1903 & Boulenger 1903 \\
\hline M. acuta Wang, Li \& Jin, 2014 & Li et al. 2014 \\
\hline M. ancrae Mahony, Teeling \& Biju, 2013 & Mahony et al. 2013 \\
\hline M. angka Wu, Suwannapoom, Poyarkov, Chen, Pawangkhanant, Xu, Jin, Murphy \& Che, 2019 & Wu et al. 2019 \\
\hline M. auralensis Ohler, Swan \& Daltry, 2002 & Ohler et al. 2002 \\
\hline M. baluensis (Boulenger, 1899) & Boulenger $1899 \mathrm{a}$ \\
\hline M. baolongensis Ye, Fei \& Xie, 2007 & Ye et al. 2007 \\
\hline M. binchuanensis Ye \& Fei, 1995 & Ye and Fei 1995 \\
\hline M. binlingensis Jiang, Fei \& Ye, 2009 & Fei et al. 2009 \\
\hline M. boettgeri (Boulenger, 1899) & Boulenger $1899 \mathrm{~b}$ \\
\hline M. brachykolos Inger \& Romer, 1961 & Inger and Romer 1961 \\
\hline M. carinense (Boulenger, 1889) & Boulenger 1889 \\
\hline M. caobangensis Nguyen, Pham, Nguyen, Luong, \& Ziegler, 2020 & Nguyen et al. 2020 \\
\hline M. caudoprocta Shen, 1994 & Shen. 1994 \\
\hline M. cheni (Wang \& Liu, 2014) & Wang et al. 2014 \\
\hline M. chishuiensis $\mathrm{Xu}, \mathrm{Li}, \mathrm{Liu}$, Wei \& Wang, 2020 & Xu et al. 2020 \\
\hline M. chuannanensis (Fei, Ye \& Huang, 2001) & Fei et al. 2001 \\
\hline M. damrei Mahony, 2011 & Mahony 2011 \\
\hline M. daweimontis Rao \& Yang, 1997 & Rao and Yang 1997 \\
\hline M. dongguanensis Wang \& Wang, 2019 & Wang et al. 2019b \\
\hline M. dringi Inger, Stuebing \& Tan, 1995 & Inger et al. 1995 \\
\hline M. edwardinae Inger, 1989 & Inger 1989 \\
\hline M. elfina Poyarkov, Duong, Orlov, Gogoleva, Vassilieva, Nguyen, Nguyen, Nguyen, Che \& Mahony, 2017 & Poyarkov et al. 2017 \\
\hline $\begin{array}{l}\text { M. fansipanensis Tapley, Cutajar, Mahony, Nguyen, Dau, Luong, Le, Nguyen, Nguyen, Portway, Luong \& } \\
\text { Rowley, } 2018\end{array}$ & Tapley et al. 2018 \\
\hline M. feae Boulenger, 1887 & Boulenger 1887 \\
\hline M. feii Yang, Wang \& Wang, 2018 & Yang et al. 2018 \\
\hline M. flavipunctata Mahony, Kamei, Teeling \& Biju, 2018 & Mahony et al. 2018 \\
\hline M. gerti (Ohler, 2003) & Ohler 2003 \\
\hline M. gigantica Liu, Hu \& Yang, 1960 & Liu et al. 1960 \\
\hline M. glandulosa Fei, Ye \& Huang, 1990 & Fei et al. 1990 \\
\hline M. hansi (Ohler, 2003) & Ohler 2003 \\
\hline M. himalayana Mahony, Kamei, Teeling \& Biju, 2018 & Mahony et al. 2018 \\
\hline $\begin{array}{l}\text { M. hoanglienensis Tapley, Cutajar, Mahony, Nguyen, Dau, Luong, Le, Nguyen, Nguyen, Portway, Luong \& } \\
\text { Rowley, } 2018\end{array}$ & Tapley et al. 2018 \\
\hline M. huangshanensis Fei \& Ye, 2005 & Fei and Ye 2005 \\
\hline M. insularis (Wang, Liu, Lyu, Zeng \& Wang, 2017) & Wang et al. $2017 \mathrm{a}$ \\
\hline M. intermedia Smith, 1921 & Smith 1921 \\
\hline M. jiangi Liu, Li, Wei, Xu, Cheng, Wang \& Wu, 2020 & Liu et al. 2020 \\
\hline M. jingdongensis Fei \& Ye, 1983 & Fei et al. 1983 \\
\hline M. jinggangensis (Wang, 2012) & Wang et al. 2012 \\
\hline M. jiulianensis Wang, Zeng, Lyu \& Wang, 2019 & Wang et al. $2019 \mathrm{~b}$ \\
\hline M. kalimantanensis Munir, Hamidy, Matsui, Iskandar, Sidik \& Shimada, 2019 & Munir et al. 2019 \\
\hline M. kobayashii Malkmus \& Matsui, 1997 & Malkmus and Matsui 1997 \\
\hline
\end{tabular}




\begin{tabular}{|c|c|}
\hline Species & Literature \\
\hline M. koui Mahony, Foley, Biju \& Teeling, 2017 & Mahony et al. 2017 \\
\hline M. kuatunensis Pope, 1929 & Pope 1929 \\
\hline M. Lancip Munir, Hamidy, Farajallah \& Smith, 2018 & Munir et al. 2018 \\
\hline M. leishanensis $\mathrm{Li}, \mathrm{Xu}$, Liu, Jiang, Wei \& Wang, 2018 & Li et al. $2018 b$ \\
\hline M. lekaguli Stuart, Chuaynkern, Chan-ard \& Inger, 2006 & Stuart et al. 2006 \\
\hline M. liboensis (Zhang, Li, Xiao, Li, Pan, Wang, Zhang \& Zhou, 2017) & Zhang et al. 2017 \\
\hline M. ligayae Taylor, 1920 & Taylor 1920 \\
\hline M. lini (Wang \& Yang, 2014) & Wang et al. 2014 \\
\hline M. lishuiensis (Wang, Liu \& Jiang, 2017) & Wang et al. $2017 \mathrm{~b}$ \\
\hline M. longipes Boulenger, 1886 & Boulenger 1886 \\
\hline M. major Boulenger, 1908 & Boulenger 1908 \\
\hline M. mangshanensis Fei \& Ye, 1990 & Fei et al. 2012 \\
\hline M. maosonensis Bourret, 1937 & Bourret 1937 \\
\hline M. medogensis Fei, Ye \& Huang, 1983 & Fei et al. 1983 \\
\hline M. megacephala Mahony, Sengupta, Kamei \& Biju, 2011 & Mahony et al. 2011 \\
\hline M. microstoma (Boulenger, 1903) & Boulenger 1903 \\
\hline M. minor Stejneger, 1926 & Stejneger 1926 \\
\hline M. mirabilis Lyu, Wang \& Zhao, 2020 & Lyu et al. 2020 \\
\hline M. montana Kuhl \& Van Hasselt, 1822 & Kuhl and Van Hasselt 1822 \\
\hline M. monticola (Günther, 1864) & Günther 1864 \\
\hline M. mufumontana Wang, Lyu \& Wang, 2019 & Wang et al. 2019b \\
\hline M. nankiangensis Liu \& Hu, 1966 & Hu and Liu 1966 \\
\hline M. nankunensis Wang, Zeng \& Wang, 2019 & Wang et al. 2019b \\
\hline M. nanlingensis Lyu, Wang, Liu \& Wang, 2019 & Wang et al. 2019b \\
\hline M. nasuta (Schlegel, 1858) & Schlegel 1858 \\
\hline M. obesa Wang, Li \& Zhao, 2014 & Wang et al. 2014 \\
\hline M. ombrophila Messenger \& Dahn, 2019 & Messenger et al. 2019 \\
\hline M. omeimontis Liu, 1950 & Liu 1950 \\
\hline M. oreocrypta Mahony, Kamei, Teeling \& Biju, 2018 & Mahony et al. 2018 \\
\hline M. oropedion Mahony, Teeling \& Biju, 2013 & Mahony et al. 2013 \\
\hline M. orientalis $\mathrm{Li}$, Lyu, Wang \& Wang, 2020 & Li et al. 2020 \\
\hline M. pachyproctus Huang, 1981 & Huang and Fei 1981 \\
\hline M. palpebralespinosa Bourret, 1937 & Bourret 1937 \\
\hline M. parallela Inger \& Iskandar, 2005 & Inger and Iskandar 2005 \\
\hline M. parva (Boulenger, 1893) & Boulenger 1893 \\
\hline M. periosa Mahony, Kamei, Teeling \& Biju, 2018 & Mahony et al. 2018 \\
\hline M. popei (Zhao, Yang, Chen, Chen \& Wang, 2014) & Zhao et al. 2014 \\
\hline M. robusta Boulenger, 1908 & Boulenger 1908 \\
\hline M. rubrimera Tapley, Cutajar, Mahony, Chung, Dau, Nguyen, Luong \& Rowley, 2017 & Tapley et al. 2017 \\
\hline M. sangzhiensis Jiang, Ye \& Fei, 2008 & Jiang et al. 2008 \\
\hline M. serchhipii (Mathew \& Sen, 2007) & Mathew and Sen 2007 \\
\hline M. shapingensis Liu, 1950 & Liu 1950 \\
\hline M. shimentaina Lyu, Liu \& Wang, 2020 & Lyu et al. 2020 \\
\hline M. shuichengensis Tian \& Sun, 1995 & Tian and Sun 1995 \\
\hline M. shunhuangensis Wang, Deng, Liu, Wu \& Liu, 2019 & Wang et al. 2019a \\
\hline M. spinata Liu \& Hu, 1973 & Hu et al. 1973 \\
\hline M. stejnegeri Taylor, 1920 & Taylor 1920 \\
\hline M. synoria (Stuart, Sok \& Neang, 2006) & Stuart et al. 2006 \\
\hline M. takensis Mahony, 2011 & Mahony 2011 \\
\hline M. tuberogranulata Shen, Mo \& Li, 2010 & Mo et al. 2010 \\
\hline M. vegrandis Mahony, Teeling, Biju, 2013 & Mahony et al. 2013 \\
\hline M. wawuensis Fei, Jiang \& Zheng, 2001 & Fei et al. 2012 \\
\hline M. wugongensis Wang, Lyu \& Wang, 2019 & Wang et al. 2019b \\
\hline M. wuliangshanensis Ye \& Fei, 1995 & Ye and Fei 1995 \\
\hline M. wushanensis Ye \& Fei, 1995 & Ye and Fei 1995 \\
\hline M. xianjuensis Wang, Wu, Peng, Shi, Lu \& Wu, 2020 & Wang et al. 2020 \\
\hline M. xiangnanensis Lyu, Zeng \& Wang, 2020 & Lyu et al. 2020 \\
\hline M. yangmingensis Lyu, Zeng \& Wang, 2020 & Lyu et al. 2020 \\
\hline M. zhangi Ye \& Fei, 1992 & Ye and Fei 2012 \\
\hline M. zunhebotoensis (Mathew \& Sen, 2007) & Mathew and Sen 2007 \\
\hline
\end{tabular}




\section{Bioacoustics analyses}

The advertisement calls of the undescribed species were recorded from the holotype specimen CIBTZ20190608017 in a stream at ambient air temperature of $20.5^{\circ} \mathrm{C}$ and air humidity of $87 \%$ in the field on 8 June 2019 in Huanglian Nature Reserve, Tongzi County, Guizhou Province, China. The advertisement calls of M. sangzhiensis were recorded from the specimen CIBSZ2012062005 in a stream at ambient air temperature of $18.5^{\circ} \mathrm{C}$ and air humidity of $85 \%$ in the field on 20 June 2012 in Sangzhi County, Hunan Province, China. The advertisement calls of M. spinata were recorded from the specimen CIBLS20190801001 in a stream at ambient air temperature of $19.0^{\circ} \mathrm{C}$ and air humidity of $85 \%$ in the field on 1 August 2019 in Leishan County, Guizhou Province, China. SONY PCM-D50 digital sound recorder was used to record within $20 \mathrm{~cm}$ of the calling individual. The sound files in wave format were resampled at 48 $\mathrm{kHz}$ with sampling depth 24 bits. The sonograms and waveforms were generated by WaveSurfer software (Sjöander and Beskow 2000) from which all parameters and characters were measured. Ambient temperature was taken by a digital hygrothermograph.

\section{Results}

\section{Phylogenetic analyses}

Aligned sequence matrix of $16 \mathrm{~S}+\mathrm{COI}$ contains $1104 \mathrm{bp}$. ML and BI trees had almost consistent topology though relationships of some lineages were unresolved (Fig. 2). In trees, the undescribed species was clustered as an independent clade and sister to a clade in comprising of M. sangzhiensis and M. spinata (Fig. 2).

Genetic distances on COI gene with uncorrected $p$-distance model between all samples of the undescribed species were below $0.2 \%$. The genetic distance between the undescribed species and its closest related species $M$. sangzhiensis was $4.3 \%$ on COI gene, which was higher or at the same level with those among many pairs of congeners, for example, $3.6 \%$ between $M$. spinata and $M$. sangzhiensis, 1.8\% between $M$. huangshanensis and $M$. boettgeri, and $4.3 \%$ between $M$. maosonensis and M. mangshanensis (Suppl. material 1: Table S1).

\section{Morphological comparisons}

In PCA for males, the total variation of the first two principal components was 63.2 $\%$. In males on the two-dimensional plots of PC1 vs. PC2, the undescribed species could be distinctly separated from M. sangzhiensis and M. spinata (Fig. 3). The results of one-way ANOVA indicated that in males, the undescribed species was significantly different from $M$. sangzhiensis and M. spinata on many morphometric characters (all $p$-values < 0.05; Table 4). More detailed descriptions of results from morphological comparisons between the undescribed species and its congeners were presented in the following sections for describing the new species. 


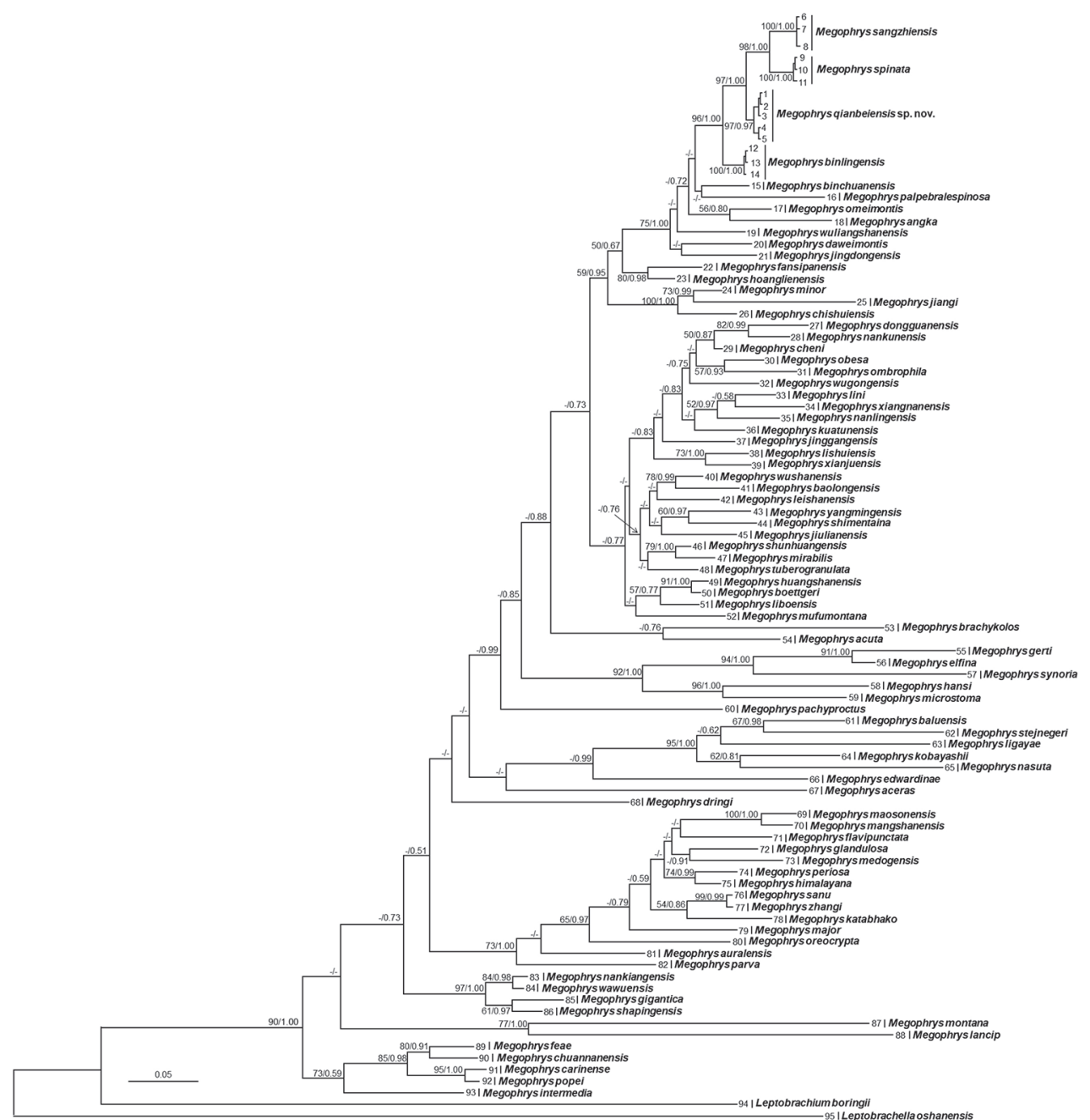

Figure 2. Bayesian Inference (BI) tree of the genus Megophrys reconstructed based on the 16S rRNA and COI gene sequences. Bayesian posterior probability resulted from BI analyses/ML bootstrap supports from Maximum Likelihood analyses were denoted beside each node. Samples 1-90 refer to Table 1.

\section{Bioacoustics comparisons}

There were many differences in sonograms and waveforms of calls between the undescribed species, M. sangzhiensis, and M. spinata (Fig. 4; Table 5). Firstly, in the note interval, the undescribed species were shorter than those of both M. sangzhiensis and M. spinata. Secondly, the dominant frequency of call in the undescribed species was lower than both of M. sangzhiensis and M. spinata. Thirdly, the amplitude of the undescribed species beginning with moderately high energy pulses, increasing slightly to a maximum by approximately mid note, and then decreasing towards the end of each note, in M. sangzhiensis beginning with maximum energy pulses and then decreasing towards the end of note, and in M. spinata beginning with lower energy pulses, then 


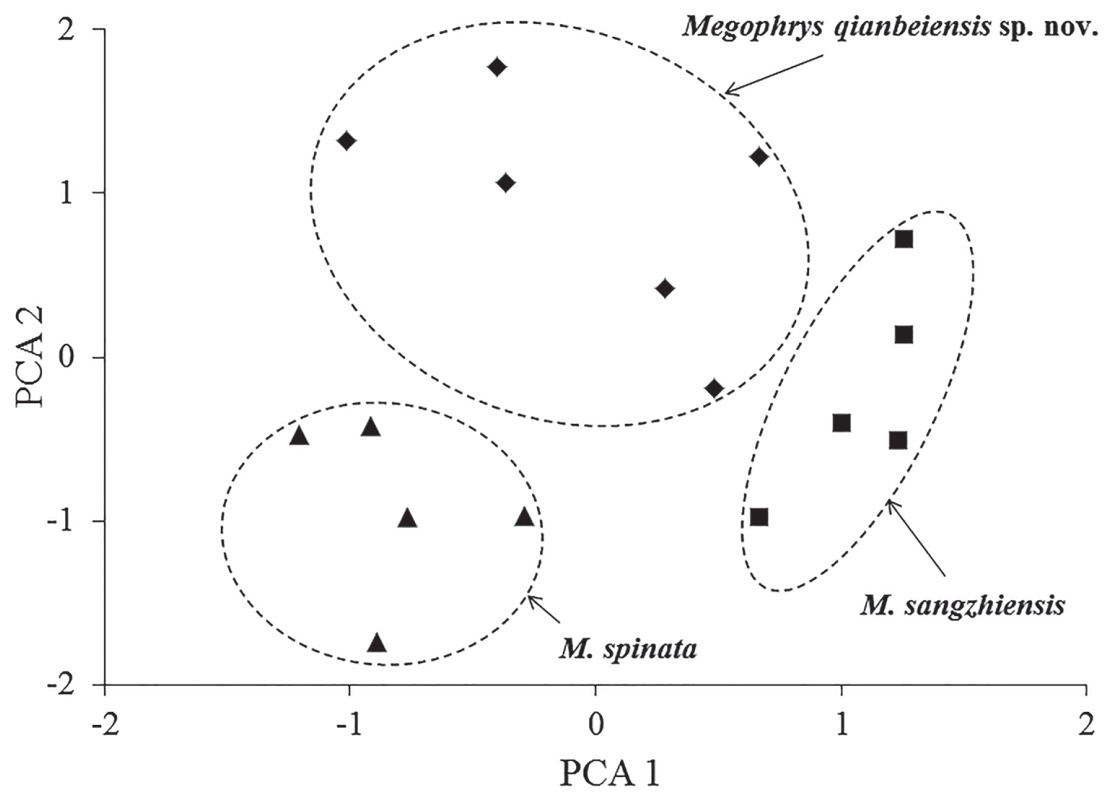

Figure 3. Plots of the first principal component (PCA1) versus the second (PCA2) for Megophrys qianbeiensis sp. nov., M. sangzhiensis, and M. spinata from principal component analyses.

Table 4. Morphometric comparisons between Megophrys qianbeiensis sp. nov., M. sangzhiensis, and M. spinata. Units given in mm. Abbreviations for the species name: MQ, Megophrys qianbeiensis sp. nov.; MSZ, M. sangzhiensis; MSP, M. spinata. See abbreviations for morphometric characters in Materials and methods section.

\begin{tabular}{|c|c|c|c|c|c|c|c|c|}
\hline & \multirow{2}{*}{\multicolumn{2}{|c|}{$\begin{array}{c}\text { Megophrys qianbeiensis sp. nov. } \\
\text { males }(\mathrm{N}=6)\end{array}$}} & \multirow{2}{*}{\multicolumn{2}{|c|}{$\begin{array}{c}\text { M. sangzhiensis } \\
\text { males }(\mathrm{N}=5)\end{array}$}} & \multirow{2}{*}{\multicolumn{2}{|c|}{$\begin{array}{c}\text { M. spinata } \\
\text { males }(\mathrm{N}=5)\end{array}$}} & \multicolumn{2}{|c|}{$p$-value from ANOVA in male } \\
\hline & & & & & & & \multirow[b]{2}{*}{ MQ vs. MSZ } & \multirow[b]{2}{*}{ MQ vs. MSP } \\
\hline & Range & Mean \pm SD & Range & Mean \pm SD & Range & Mean \pm SD & & \\
\hline SVL & $49.3-58.2$ & $54.3 \pm 3.09$ & $56.1-59.8$ & $58.3 \pm 1.56$ & $51.2-56.2$ & $53.9 \pm 1.84$ & 0.029 & 0.851 \\
\hline HDL & $14.6-17.0$ & $15.6 \pm 0.93$ & $16.1-18.0$ & $17.2 \pm 0.96$ & $14.3-15.8$ & $14.8 \pm 0.59$ & 0.027 & 0.124 \\
\hline HDW & $18.3-21.0$ & $19.9 \pm 1.08$ & $20.0-21.5$ & $20.8 \pm 0.55$ & $18.4-19.1$ & $18.7 \pm 0.30$ & 0.123 & 0.037 \\
\hline SL & $6.7-7.2$ & $6.9 \pm 0.17$ & $6.6-8.2$ & $7.4 \pm 0.58$ & $5.7-7.1$ & $6.4 \pm 0.56$ & 0.067 & 0.085 \\
\hline TYD & $3.2-4.3$ & $3.5 \pm 0.39$ & $3.1-3.7$ & $3.4 \pm 0.21$ & $2.5-2.9$ & $2.7 \pm 0.15$ & 0.639 & 0.001 \\
\hline IFE & $7.8-10.9$ & $9.7 \pm 1.13$ & $9.4-10.9$ & $10.2 \pm 0.54$ & $8.5-9.6$ & $9.1 \pm 0.39$ & 0.340 & 0.348 \\
\hline IAE & $14.0-16.4$ & $15.5 \pm 0.86$ & $16.1-17.3$ & $16.8 \pm 0.62$ & $14.0-14.4$ & $14.2 \pm 0.18$ & 0.019 & 0.009 \\
\hline NED & $2.4-3.4$ & $2.9 \pm 0.36$ & $3.0-3.9$ & $3.4 \pm 0.36$ & $2.7-3.0$ & $2.8 \pm 0.11$ & 0.060 & 0.618 \\
\hline NSD & $3.5-4.3$ & $3.8 \pm 0.29$ & $3.7-4.6$ & $4.2 \pm 0.34$ & $3.4-4.1$ & $3.8 \pm 0.27$ & 0.101 & 0.683 \\
\hline IND & $5.7-7.5$ & $6.7 \pm 0.62$ & $6.8-7.7$ & $7.2 \pm 0.34$ & $5.8-6.3$ & $6.0 \pm 0.18$ & 0.150 & 0.056 \\
\hline IOD & $3.7-5.3$ & $4.5 \pm 0.59$ & $4.7-5.8$ & $5.1 \pm 0.45$ & $4.2-5.3$ & $4.9 \pm 0.44$ & 0.117 & 0.257 \\
\hline ED & $5.4-6.9$ & $6.3 \pm 0.58$ & $6.1-7.4$ & $6.7 \pm 0.51$ & $5.1-6.0$ & $5.6 \pm 0.34$ & 0.280 & 0.041 \\
\hline UEW & $5.1-6.2$ & $5.6 \pm 0.43$ & $5.1-6.1$ & $5.7 \pm 0.42$ & $4.5-6.1$ & $5.0 \pm 0.65$ & 0.484 & 0.126 \\
\hline LAL & $20.4-25.4$ & $24.0 \pm 1.84$ & $26.2-28.2$ & $26.9 \pm 0.78$ & $24.0-25.1$ & $24.3 \pm 0.44$ & 0.014 & 0.654 \\
\hline LW & $5.7-7.4$ & $6.6 \pm 0.55$ & $6.1-6.7$ & $6.4 \pm 0.24$ & $5.5-7.4$ & $6.3 \pm 0.72$ & 0.394 & 0.364 \\
\hline HLL & $76.9-93.7$ & $87.8 \pm 6.07$ & $97.3-105.0$ & $100.3 \pm 2.84$ & $85.7-99.0$ & $91.8 \pm 5.27$ & 0.003 & 0.280 \\
\hline THL & $24.1-28.4$ & $26.6 \pm 1.82$ & $27.9-31.6$ & $29.6 \pm 1.59$ & $26.1-29.8$ & $27.6 \pm 1.38$ & 0.019 & 0.343 \\
\hline TL & $25.0-32.4$ & $29.2 \pm 2.63$ & $31.6-32.6$ & $32.2 \pm 0.42$ & $28.9-30.4$ & $29.6 \pm 0.63$ & 0.038 & 0.713 \\
\hline TW & $7.0-8.7$ & $8.0 \pm 0.58$ & $7.3-8.1$ & $7.7 \pm 0.32$ & $6.0-8.0$ & $7.0 \pm 0.91$ & 0.404 & 0.053 \\
\hline TFL & $34.5-44.0$ & $40.4 \pm 3.58$ & $42.9-46.1$ & $44.2 \pm 1.32$ & $39.2-43.1$ & $40.9 \pm 1.55$ & 0.062 & 0.761 \\
\hline FL & $24.5-29.4$ & $27.5 \pm 1.95$ & $26.7-29.9$ & $28.9 \pm 1.28$ & $26.4-29.6$ & $28.1 \pm 1.16$ & 0.201 & 0.531 \\
\hline
\end{tabular}



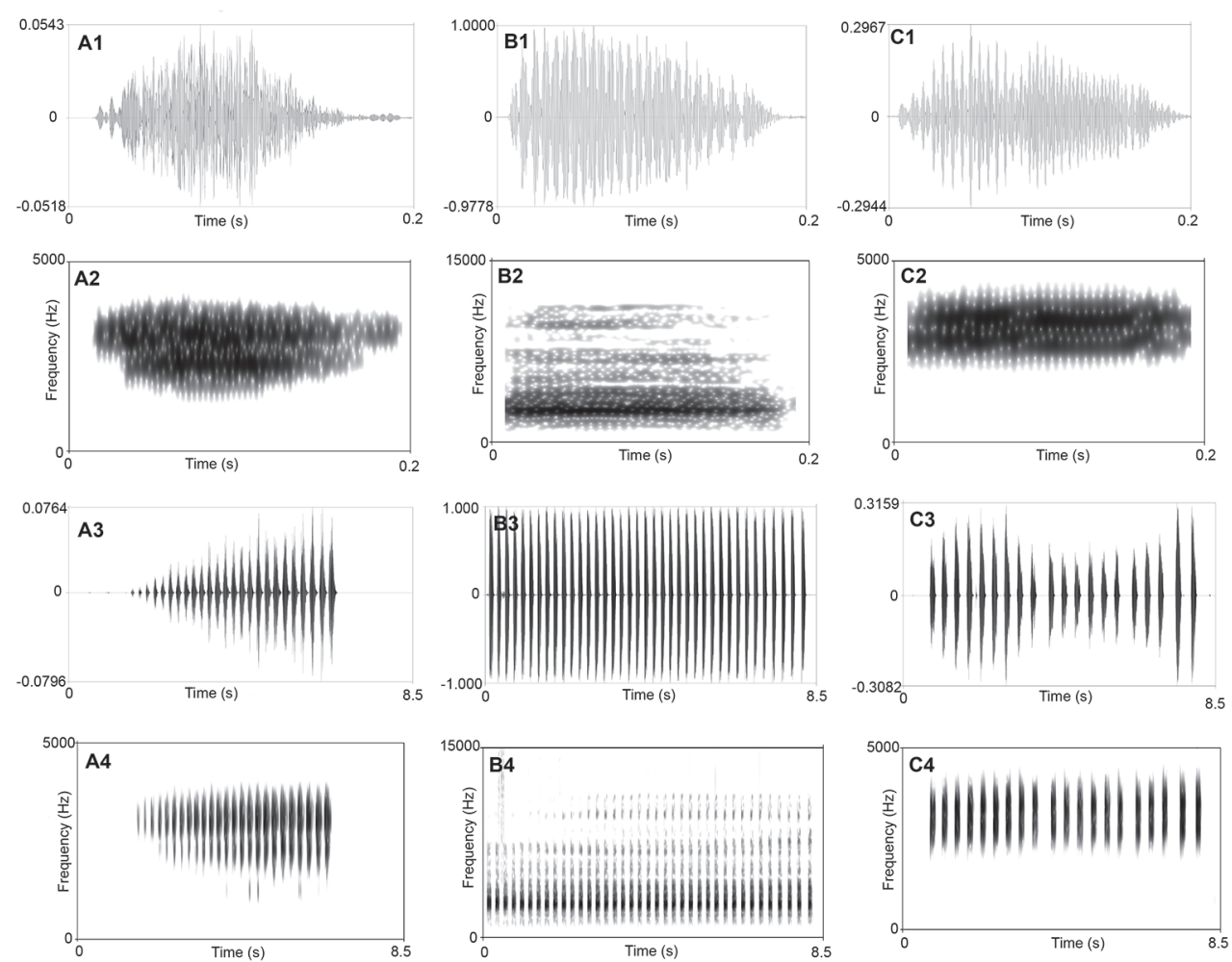

Figure 4. Visualization of advertisement calls of Megophrys qianbeiensis sp. nov., M. sangzhiensis, and $M$. spinata Al waveform showing one note of Megophrys qianbeiensis sp. nov. A2 sonogram showing one note of Megophrys qianbeiensis sp. nov. A3 waveform showing 25 notes of one call of Megophrys qianbeiensis sp. nov. A4 sonogram showing 25 notes of one call of Megophrys qianbeiensis sp. nov. B I waveform showing one note of $M$. sangzhiensis B2 sonogram showing one note of $M$. sangzhiensis B3 waveform showing 38 notes of one call of $M$. sangzhiensis. B4 sonogram showing 38 notes of one call of $M$. sangzhiensis $\mathbf{C l}$ waveform showing one note of $M$. spinata $\mathbf{C 2}$ sonogram showing one note of $M$. spinata $\mathbf{C} 3$ waveform showing 20 notes of one call of $M$. spinata $\mathbf{C} 4$ sonogram showing 20 notes of one call of $M$. spinata.

Table 5. Comparisons of characteristics of advertisement calls of Megophrys qianbeiensis sp. nov., M. sangzhiensis, and M. spinata.

\begin{tabular}{lcccccc}
\hline \multirow{2}{*}{ Call character } & \multicolumn{2}{c}{ Megophrys qianbeiensis sp. nov. } & \multicolumn{2}{c}{ M. sangzhiensis } & \multicolumn{2}{c}{ M. spinata } \\
\cline { 2 - 7 } & Range & Mean \pm SD & Range & Mean \pm SD & Range & Mean \pm SD \\
\hline Number of notes in a call & $14-26$ & $22.5 \pm 4.4$ & 38 & $/$ & $7-28$ & $17 \pm 7.92$ \\
Call duration (ms) & $2832-5621$ & $4413 \pm 972$ & 8152 & $/$ & $1500-6623$ & $3905 \pm 2010$ \\
Call interval (ms) & $6812-14387$ & $10878 \pm 2701$ & $/$ & $/$ & $592-5770$ & $2708 \pm 1863.33$ \\
Note duration (ms) & $129-211$ & $167 \pm 0.02$ & $107-155$ & $120.3 \pm 8.73$ & $107-123$ & $114 \pm 3.79$ \\
Note interval (ms) & $34-94$ & $57 \pm 0.01$ & $72-132$ & $95.6 \pm 13.89$ & $113-232$ & $147 \pm 33.12$ \\
Dominant frequency $(\mathrm{Hz})$ & $2250-3000$ & $2469 \pm 197.47$ & $10380-13200$ & $11795 \pm 670.58$ & $4260-4589$ & $4416 \pm 130.04$ \\
Temperature $\left({ }^{\circ} \mathrm{C}\right)$ & 20.5 & & 18.5 & & 19.0 & \\
\hline
\end{tabular}


increasing to the maximum by approximately one-four note and then decreasing to the mid note then increasing to the second highest energy pulses and then decreasing towards the end of note.

\section{Taxonomic accounts}

\section{Megophrys qianbeiensis sp. nov.}

http://zoobank.org/C6C89A51-8178-4C7B-A100-80C0D2D42AD3

Figures 4A1-C1, 5, 6A1-A6, 7; Tables 1, 2, 4, 5, Suppl. material 2: Table S2

Type material. Holotype. CIBTZ20190608017 (Figs 5, 6), adult male, from Huanglian Nature Reserve, Tongzi County, Guizhou Province, China $\left(28.498056^{\circ} \mathrm{N}\right.$, 107.046944 E, ca. 1500 m a.s.l.), collected by Shi-Ze Li 8 June 2019.

Paratype. Four adult males from the same place as holotype, and one from Kuankuoshui National Nature Reserve $\left(28.21835^{\circ} \mathrm{N}, 107.166388^{\circ} \mathrm{E}\right.$, ca. $1520 \mathrm{~m}$ a.s.l.) collected by Shi-Ze Li. CIBKKS20180722001 collected 22 July 2018 from Kuankuoshui National Nature Reserve and CIBTZ20160715003 collected 15 July 2016, CIBTZ20190608015, CIBTZ20190608016 and CIBTZ20190608018 collected 8 June 2019 from Kuankuoshui National Nature Reserve.

Diagnosis. Megophrys qianbeiensis sp. nov. is assigned to the genus Megophrys based on molecular phylogenetic analyses and the following generic diagnostic characters: snout shield-like; projecting beyond the lower jaw; canthus rostralis distinct; chest glands small and round, closer to the axilla than to midventral line; femoral glands on rear part of thigh; vertical pupils.

Megophrys qianbeiensis sp. nov. could be distinguished from its congeners by a combination of the following morphological characters: body size moderate (SVL $49.3-58.2 \mathrm{~mm}$ in males); vomerine ridges present distinctly, vomerine teeth present; tongue feebly notched behind; tympanum distinctly visible, oval; two metacarpal tubercles in hand; toes with one-third webbing and wide lateral fringes; heels overlapped when thighs are positioned at right angles to the body; tibiotarsal articulation reaching the level between tympanum and eye when leg stretched forward; an internal single subgular vocal sac present in male; in breeding male, the nuptial pads with large and sparse black nuptial spines present on the dorsal bases of the first two fingers.

Description of holotype. (Figs 5, 6). SVL $56.3 \mathrm{~mm}$; head width larger than head length (HDW/HDL ratio ca. 1.3); snout obtusely pointed, protruding well beyond the margin of the lower jaw in ventral view; loreal region vertical and concave; canthus rostralis well-developed; top of head flat in dorsal view; eye large, eye diameter $44.5 \%$ of head length; pupils vertical; nostril orientated laterally, closer to snout than eye; tympanum distinct, TYP/EYE ratio 0.49 ; vomerine ridges present distinctly as V-shape, vomerine teeth present; margin of tongue smooth, feebly notched behind.

Forelimbs slender, the length of lower arm and hand $42.6 \%$ of SVL; fingers burly, relative finger lengths: II $<$ I $<$ V $<$ III; tips of digits globular, without lateral fringes; subarticular tubercle distinct at the base of each finger; two metacarpal tubercles, prominent, oval-shaped, the inner one bigger than the outer one. 


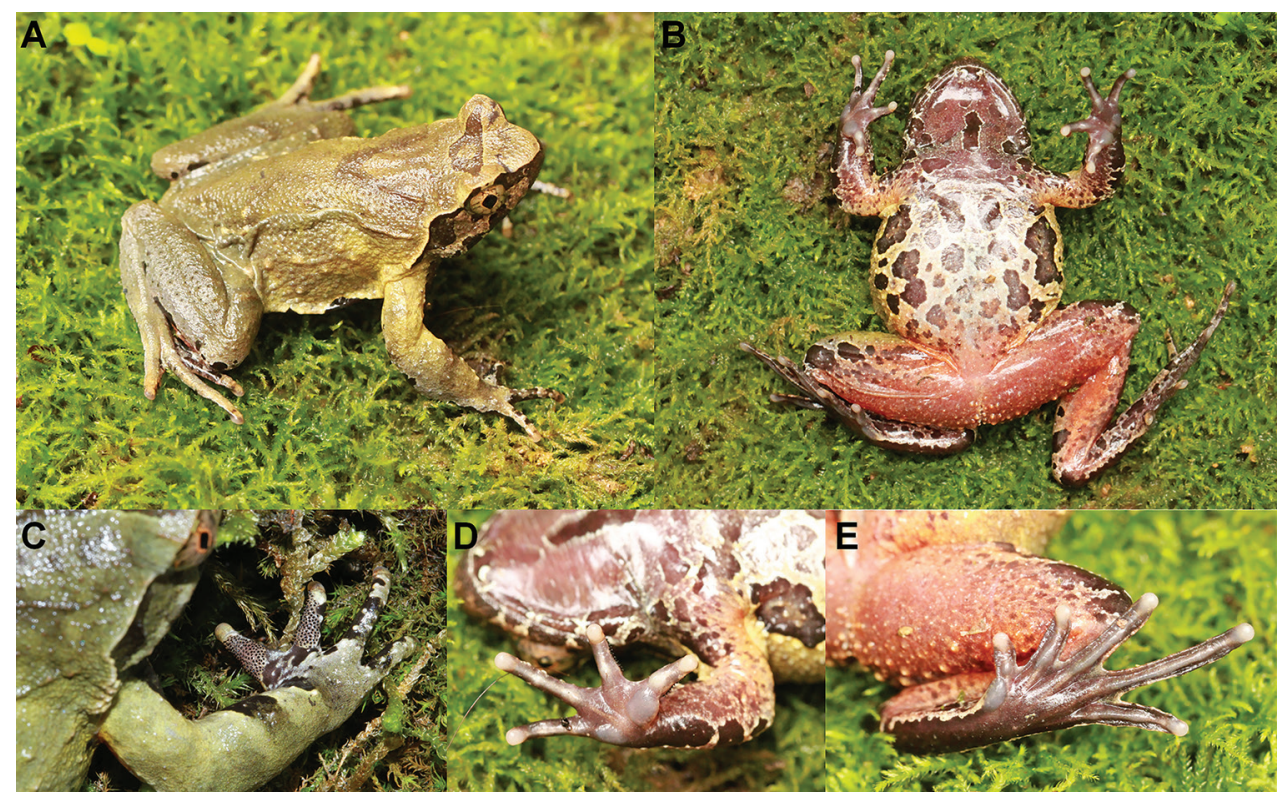

Figure 5. Photos of the holotype CIBTZ20190608017 of Megophrys qianbeiensis sp. nov. in life A dorsal view $\mathbf{B}$ ventral view $\mathbf{C}$ dorsal view of hand $\mathbf{D}$ ventral view of hand $\mathbf{E}$ ventral view of foot.

Hindlimbs slender, 1.54 times of SVL; heels overlapping when thighs are positioned at right angles to the body, tibiotarsal articulation reaching tympanum to eye when leg stretched forward; tibia length longer than thigh length; relative toe lengths I $<$ II $<$ V $<$ III $<$ IV; tips of toes round, slightly dilated; subarticular tubercles present on the base of each toes ; toes with one-third webbing and relative wide lateral fringe; inner metatarsal tubercle oval-shaped; outer metatarsal tubercle absent.

Dorsal skin rough, with numerous granules with black spins; several large warts scattered on flanks; tubercles on the dorsum forming a weak V-shaped ridge; two discontinuous dorsolateral parallel ridges on either side of the V-shaped ridges; an inverted triangular brown speckle between two upper eyelids; several tubercles on the flanks and dorsal surface of thighs and tibias; supratympanic fold distinct.

Ventral surface smooth with numerous white granules; glands on chest indistinct; femoral glands on rear of thighs, numerous white granules on outer thighs; posterior end of the body distinctly protruding and forming an arc-shaped swelling above the anal region.

Coloration of holotype in life (Fig. 5). An inverted triangular brown speckle between the eyes; V-shaped ridges on the dorsum with brown speckle, on transverse bands on the dorsal surface of the thigh and shank; several dark brown and white vertical bars on the lower and upper lip; belly whitish grey with dark brown marbling; ventral surface of posterior limb orange with numerous granules; palms, soles and tip of digits uniform purple grey; femoral glands white.

Coloration of holotype in preservation (Fig. 6). Color of dorsal surface fades to brownness; the inverted triangular brown speckle between the eyes and V-shaped ridges on dorsum indistinct; ventral surface greyish white; creamy-white substitutes 

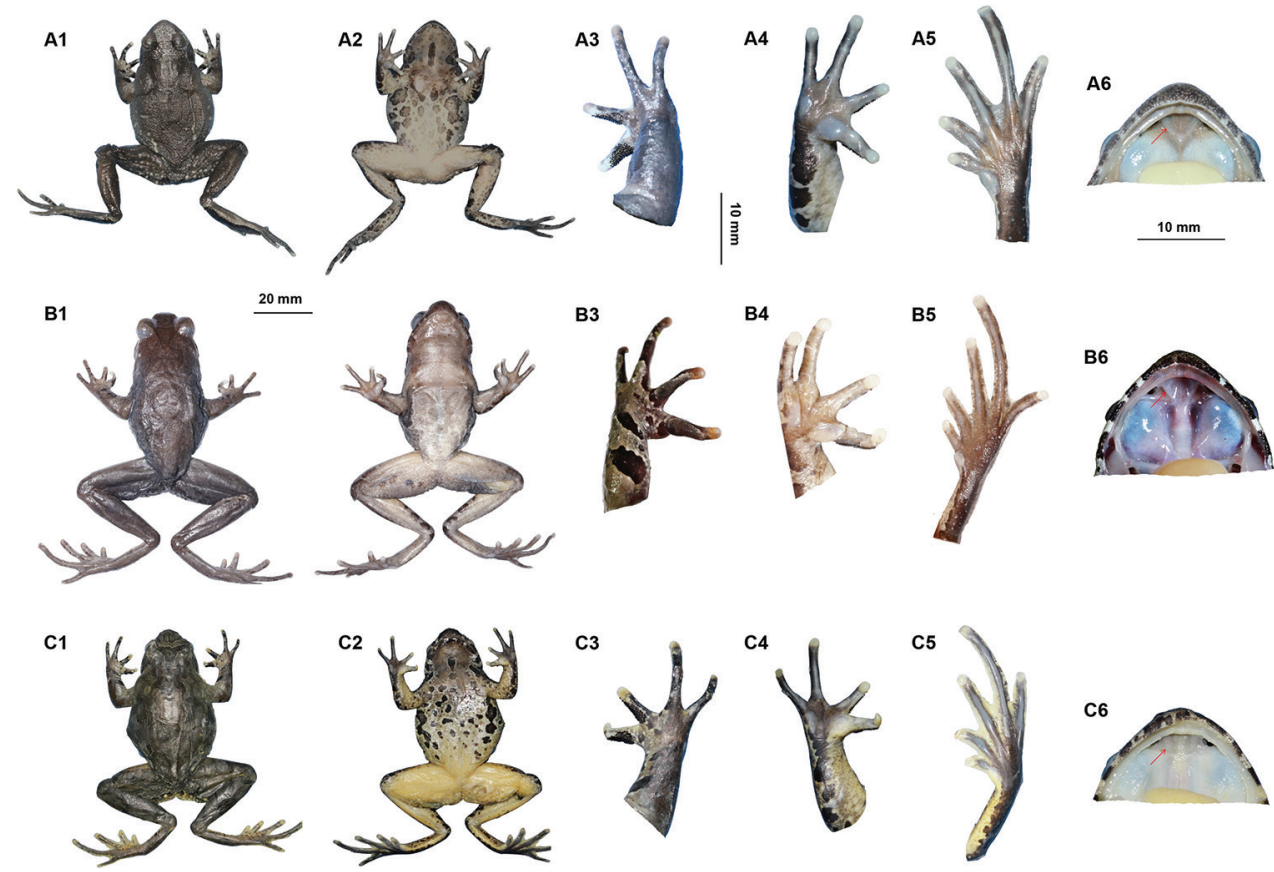

Figure 6. Photos of specimens of Megophrys qianbeiensis sp. nov., M. sangzhiensis and M. spinata Al-A6 dorsal view, ventral view, dorsal view of hand, ventral view of hand, ventral view of foot and view of oral cavity of the holotype specimen CIBTZ20190608017 of Megophrys qianbeiensis sp. nov. B I-B6 dorsal view, ventral view, dorsal view of hand, ventral view of hand, ventral view of foot and view of oral cavity of CIBSZ2012062005 of $M$. sangzhiensis $\mathbf{C I}-\mathbf{C 6}$ dorsal view, ventral view, dorsal view of hand, ventral view of hand, ventral view of foot and view of oral cavity of CIBLS20190801001 of M. spinata. Arrow point to vomerine ridge.

the purple grey on tip of digits; the posterior of ventral surface of body, inner of thigh and upper of tibia fades to creamy-white.

Variation. In CIBTZ20160715003 the dorsolateral parallel ridges are short, just a little bit above the shoulder (Fig. 7A); in CIBTZ20190608015 the X-shaped marking on back of trunk consists of a ridge with brown spots (Fig. 7B) and the posterior belly are orange with black spots on the flank belly (Fig. 7C); in CIBKKS20180722001 the belly is grey brown with some white spots (Fig. 7D).

Advertisement call. The call description is based on recordings of the holotype CIBTZ20190608017 (Fig. 4) from the shrub leaf near the streamlet, and the ambient air temperature was $20.5^{\circ} \mathrm{C}$. Each call consists of 14-26 (mean $22.5 \pm 4.4, N=6$ ) notes. Call duration was $2832-5621 \mathrm{~ms}$ (mean $4413 \pm 972, N=6$ ). Call interval was 6812-14387 ms (mean $10878 \pm 2701, N=5$ ). Each note had a duration of 129-211 ms (mean $167 \pm 0.02, N=135$ ) and the intervals between notes 34-94 ms (mean $57 \pm 0.01, N=128$ ). Amplitude modulation within note was apparent, beginning with moderately high energy pulses, increasing slightly to a maximum by approximately mid note, and then decreasing towards the end of each note. The average dominant frequency was $2469 \pm 197.47(2250-3000 \mathrm{~Hz}, N=6)$. 


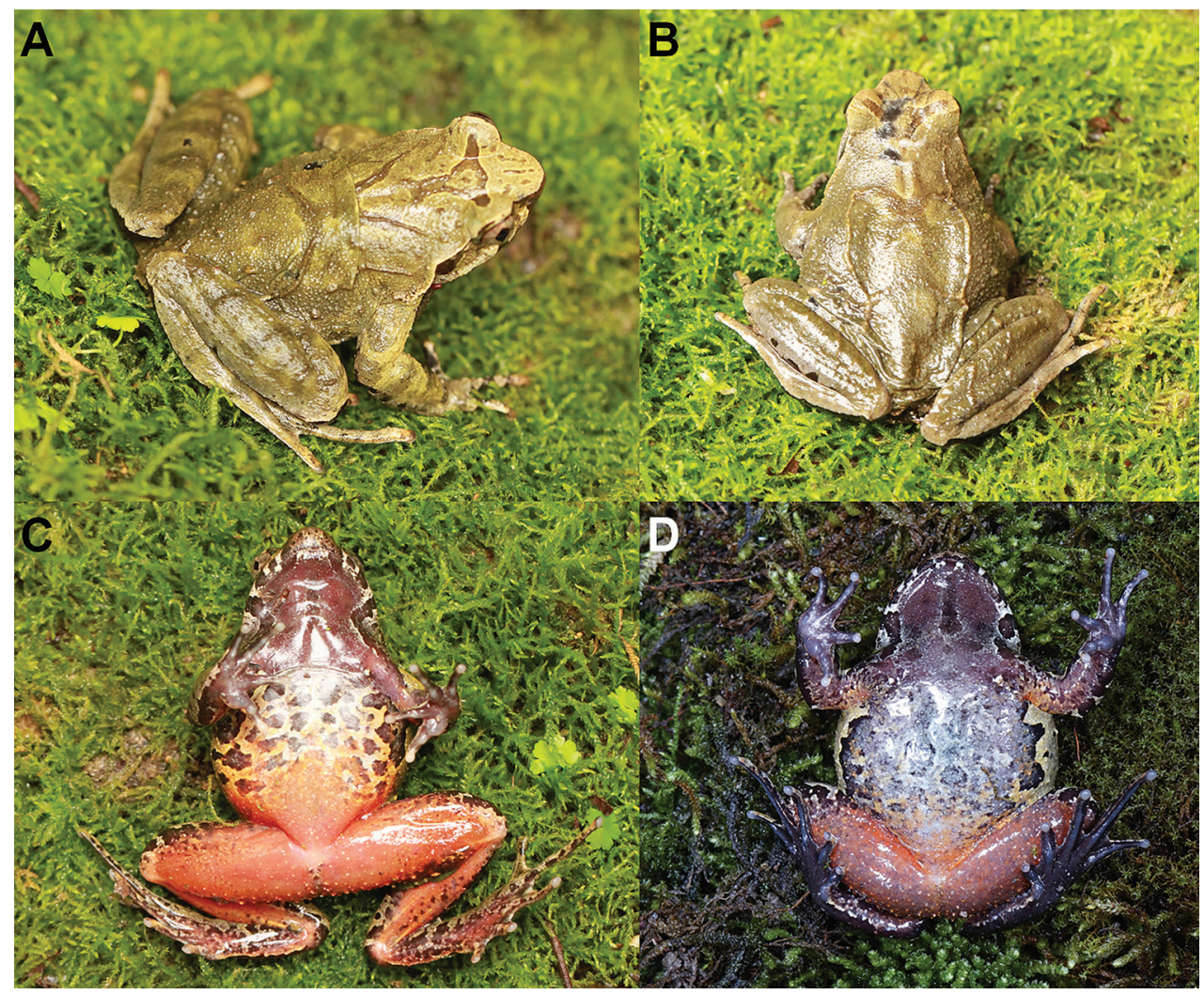

Figure 7. Color variation in Megophrys qianbeiensis sp. nov. A dorsolateral view of the specimen CIBTZ20160715003 B dorsolateral view of the specimen CIBTZ20190608015 C ventral view of the male specimen CIBTZ20190608015 D ventral view of the specimen CIBKKS20180722001.

Secondary sexual characters. Adult males have a single subgular vocal sac. In breeding males, brownish red nuptial pads are present on the dorsal bases of the first two fingers with big and sparse black nuptial spines (Fig. 5A).

Comparisons. By having moderate body size (minimum SVL $>49.8 \mathrm{~mm}$ in males), Megophrys qianbeiensis sp. nov. differs from $M$. aceras, M. acuta, M. angka, $M$. ancrae, $M$. baluensis, $M$. baolongensis, $M$. binchuanensis, $M$. binlingensis, $M$. boettgeri, M. brachykolos, $M$. caobangensis, $M$. cheni, M. daweimontis, M. dongguanensis, M. dringi, M. edwardinae, M. elfina, $M$. fansipanensis, M. feii, M. gerti, $M$. hansi, $M$. hoanglienensis, $M$. huangshanensis, $M$. insularis, $M$. jiangi, $M$. jinggangensis, $M$. jiulianensis, M. kuatunensis, M. lancip, M. leishanensis, M. lini, M. lishuiensis, M. longipes, M. major, M. microstoma, M. minor, M. monticola, M. mufumontana, M. nankunensis, M. nanlingensis, M. obesa, M. ombrophila, M. oropedion, M. pachyproctus, $M$. palpebralespinosa, M. parallela, M. parva, M. rubrimera, M. serchhipii, M. shimentaina, $M$. shunhuangensis, $M$. tuberogranulata, $M$. vegrandis, $M$. wawuensis, $M$. wugongensis, M. wuliangshanensis, $M$. wushanensis, $M$. xianjuensis, M. zhangi, M. zunhebotoensis, M. xiangnanensis, and $M$. yangmingensis (vs. minimum SVL $<48.0 \mathrm{~mm}$ ). 
By having moderate body size (minimum SVL $<59.0 \mathrm{~mm}$ in males), Megophrys qianbeiensis sp. nov. differs from $M$. auralensis, $M$. carinense, $M$. caudoprocta, $M$. caudoprocta, M. chuannanensis, $M$. feae, M. gigantica, M. glandulosa, M. himalayana, M. kalimantanensis, M. kobayashii, M. ligayae, $M$. mangshanensis, $M$. orientalis, $M$. periosa, $M$. platyparietus, $M$. popei, $M$. shapingensis, and $M$. shuichengensis (vs. minimum SVL $>60.0 \mathrm{~mm}$ ).

By having vomerine teeth, Megophrys qianbeiensis sp. nov. differs from $M$. aceras, $M$. acuta, $M$. angka, $M$. auralensis, $M$. baolongensis, $M$. binchuanensis, $M$. binlingensis, $M$. boettgeri, M. brachykolos, $M$. caobangensis, $M$. cheni, M. chishuiensis, $M$. dringi, $M$. jiangi, M. leishanensis, M. lini, M. lishuiensis, M. major, M. microstoma, M. minor,. M. mirabilis, M. mufumontana, M. nankiangensis, M. obesa, M. ombrophila, $M$. shapingensis, $M$. shuichengensis, $M$. shunhuangensis, $M$. tuberogranulata, $M$. vegrandis, M. wawuensis, M. wugongensis, $M$. wuliangshanensis, $M$. wushanensis, $M$. xianjuensis, M. xiangnanensis, and M. yangmingensis (vs. absent).

By the absence of horn-like tubercle at the edge of each upper eyelid, Megophrys qianbeiensis sp. nov. differs from $M$. aceras, $M$. acuta, $M$. angka, $M$. ancrae, $M$. auralensis, $M$. baluensis, $M$. baolongensis, $M$. boettgeri, $M$. brachykolos, $M$. caobangensis, $M$. carinense, $M$. caudoprocta, $M$. cheni, $M$. chishuiensis, $M$. chuannanensis, $M$. daweimontis, $M$. dongguanensis, $M$. dringi, $M$. edwardinae, $M$. elfina, $M$. fansipanensis, M. feae, M. feii, M. flavipunctata, M. gerti, M. glandulosa, M. hansi, M. himalayana, $M$. hoanglienensis, $M$. huangshanensis, $M$. insularis, $M$. intermedia, $M$. jiangi, $M$. jingdongensis, $M$. jinggangensis, $M$. jiulianensis, M. kalimantanensis, M. koui, M. kuatunensis, M. lancip, M. leishanensis, M. lekaguli, M. liboensis, M. ligayae, M. lini, $M$. lishuiensis, $M$. longipes, $M$. mangshanensis, $M$. medogensis, $M$. microstoma, $M$. mirabilis, $M$. montana, $M$. mufumontana, $M$. nankunensis, $M$. nanlingensis, $M$. nasuta, M. obesa, M. ombrophila, $M$. omeimontis, $M$. oreocrypta, $M$. orientalis, $M$. palpebralespinosa, M. parallela, M. parva, M. periosa, M. platyparietus, M. popei, M. rubrimera, $M$. shimentaina, $M$. shuichengensis, $M$. shunhuangensis, $M$. stejnegeri, $M$. synoria, $M$. vegrandis, $M$. wugongensis, $M$. xianjuensis, $M$. xiangnanensis, and $M$. yangmingensis (vs. present).

With the tongue feebly notched behind, Megophrys qianbeiensis sp. nov. differs from $M$. aceras, $M$. acuta, $M$. angka, $M$. auralensis, $M$. brachykolos, $M$. caobangensis, $M$. caudoprocta, $M$. dongguanensis, $M$. elfina, $M$. hansi, $M$. jiangi, $M$. jinggangensis, $M$. lancip, M. leishanensis, M. lekaguli, M. lini, M. lishuiensis, M. megacephala, M. mufumontana, $M$. nankunensis, $M$. obesa, $M$. ombrophila, $M$. orientalis, $M$. palpebralespinosa, $M$. parallela, $M$. parva, M. shunhuangensis, $M$. takensis, $M$. wushanensis, and $M$. xianjuensis (vs. tongue not notched behind in the latter), and differs from $M$. cheni, $M$. damrei, M. dringi, M. flavipunctata, M. gigantica, and M. popei (vs. tongue notched behind).

By having lateral wide fringes on toes, Megophrys qianbeiensis sp. nov. differs from $M$. angka, $M$. baolongensis, $M$. brachykolos, $M$. caobangensis, $M$. damrei, $M$. daweimontis, $M$. dongguanensis, $M$. fansipanensis, $M$. feae, M. himalayana, M. hoanglienensis, $M$. huangshanensis, $M$. insularis, $M$. jiangi, $M$. jiulianensis, $M$. kalimantanensis, $M$. koui, $M$. leishanensis, M. lekaguli, $M$. lishuiensis, M. major, $M$. mangshanensis, $M$. medogensis, $M$. megacephala, $M$. microstoma, $M$. minor, $M$. nankunensis, $M$. obesa, $M$. ombrophila, $M$. oreocrypta, $M$. oropedion, $M$. pachyproctus, $M$. parva, $M$. periosa, $M$. shunhuangensis, 
M. takensis, M. tuberogranulata, $M$. wawuensis, M. wugongensis, M. wuliangshanensis, and $M$. xianjuensis (vs. lacking lateral fringes on toes).

By toes with one-third webs, Megophrys qianbeiensis sp. nov. differs from $M$. aceras, $M$. acuta, $M$. angka, $M$. auralensis, $M$. baluensis, $M$. baolongensis, $M$. binchuanensis, $M$. binlingensis, $M$. boettgeri, $M$. brachykolos, $M$. caobangensis, $M$. caudoprocta, $M$. cheni, $M$. chuannanensis, $M$. damrei, $M$. daweimontis, $M$. dongguanensis, $M$. dringi, M. elfina, M. fansipanensis, M. feae, M. feii, M. flavipunctata, M. gerti, M. gigantica, M. glandulosa, M. hansi, M. hoanglienensis, M. huangshanensis, M. insularis, $M$. jiangi, $M$. jinggangensis, $M$. jiulianensis, M. kalimantanensis, M. koui, M. kuatunensis, M. lancip, M. leishanensis, M. lekaguli, M. liboensis, M. lini, M. lishuiensis, M. longipes, M. major, $M$. mangshanensis, $M$. medogensis, $M$. medogensis, $M$. megacephala, $M$. microstoma, $M$. minor, $M$. mufumontana, $M$. nankiangensis, $M$. nankunensis, $M$. nanlingensis, M. obesa, M. ombrophila, M. omeimontis, M. oropedion, M. pachyproctus, M. parva, $M$. periosa, $M$. robusta, M. rubrimera, $M$. serchhipii, $M$. shunhuangensis, $M$. takensis, $M$. tuberogranulata, $M$. vegrandis, $M$. wawuensis, $M$. wugongensis, $M$. wuliangshanensis, M. wushanensis, $M$. xianjuensis, and $M$. zhangi (vs. with rudimentary or without webs).

By heels overlapping when thighs are positioned at right angles to the body, Megophrys qianbeiensis sp. nov. differs from $M$. acuta, M. brachykolos, $M$. dongguanensis, M. huangshanensis, M. kuatunensis, M. nankunensis, M. obesa, M. ombrophila, and M. wugongensis (vs. not meeting).

By the tibiotarsal articulation reaching to the level between tympanum and eye when leg stretched forward, Megophrys qianbeiensis sp. nov. differs from $M$. daweimontis, M. glandulosa, M. lini, M. major, M. medogensis, and M. obesa (vs. reaching the anterior corner of the eye or beyond eye or nostril and tip of snout).

By having an internal single subgular vocal sac in male, Megophrys qianbeiensis sp. nov. differs from $M$. caudoprocta, $M$. shapingensis, and $M$. shuichengensis (vs. vocal sac absent).

The congeners $M$. carinense and $M$. jiangi have sympatric distribution with Megophrys qianbeiensis sp. nov. (Fei et al. 2012). The new species can be distinguished from these species by a series of morphological characters as follows. The new species differs from $M$. carinense in the smaller body size in the new species (adult males with 49.3-58.2 mm vs. adult males with $92-123 \mathrm{~mm}$ in the latter), a horn-like tubercle at the edge of each upper eyelid absent (vs. prominent in the latter), the tongue feebly notched behind (vs. notched behind in the latter). The new species differs from $M$. jiangi by a larger body size (49.3-58.2 $\mathrm{mm}$ in males in the new species vs. 34.4$39.2 \mathrm{~mm}$ in the latter), a horn-like tubercle at the edge of each upper eyelid absent (vs. present in the latter), the tongue feebly notched behind (vs. notched behind in the latter), presence of wide lateral fringes on the toes (vs. lacking in the latter), and toes with one-third webbing (vs. rudimentary webbing in the latter).

Megophrys qianbeiensis sp. nov. is phylogenetically closest to $M$. sangzhiensis and M. spinata. The new species differs from $M$. sangzhiensis by the following characters: horn-like tubercle absent at the edge of each upper eyelid (vs. present in the latter), toes with one-third webs (vs. with rudimentary webbing in the latter), vomerine ridges present distinctly as $\mathrm{V}$-shape and vomerine teeth present (vs. vomerine ridges separated and weak, vomerine teeth absent in the latter), tibiotarsal articulation reaching to the 
level between tympanum and eye when leg stretched forward (vs. reaching the anterior corner of eye in the latter), spines on nuptial pads on the first two fingers larger and sparser (vs. finer and thicker in the latter), and having significantly higher ratios of HDL, LAL, HLL, TL, and IAE to SVL. On bioacoustics, the new species differs from M. sangzhiensis in the following characters: lower dominant frequency $(2250-3000 \mathrm{~Hz}$ in the new species vs. $10380-13200 \mathrm{~Hz}$ in the latter), the amplitude beginning with moderately high energy pulses, increasing slightly to a maximum by approximately mid note, and then decreasing towards the end of each note (vs. beginning with maximum energy pulses and then decreasing towards the end of note in the latter).

The new species differs from M. spinata by the following characters: tibiotarsal articulation reaching the level between tympanum to eye when leg stretched forward (vs. reaching the anterior corner of eye in the latter), present distinctly as V-shape and vomerine teeth present (vs. vomerine ridges separated and weak, vomerine teeth absent in the latter), spines on nuptial pads on the first two fingers little weaker (vs. spines larger in the latter), and having significantly higher ratios of HDW, ED, LAL, TYD and IAE to SVL. On bioacoustics, the new species differs from $M$. spinata in the following characters: lower dominant frequency $(2250-3000 \mathrm{~Hz}$ in the new species vs. $4260-4589 \mathrm{~Hz}$ in the latter), the amplitude beginning with moderately high energy pulses, increasing slightly to a maximum by approximately mid note, and then decreasing towards the end of each note (vs. beginning with lower energy pulses, then increasing to the maximum by approximately one-four note and then decreasing to the mid note then increasing to the second highest energy pulses and then decreasing towards the end of note in the latter).

Distribution and habitats. Megophrys qianbeiensis sp. nov. is known from Huanglian Nature Reserve, Tongzi County and Kuankuoshui National Nature Reserve, Suiyang County, Guizhou Province, China at elevations between 1400-1600 m. The individuals of the new species were frequently found on stone in the streams surrounded by evergreen broadleaved forests (Fig. 8), and three sympatric amphibian species were found, i.e., Megophrys jiangi, Odorrana margaratae (Liu, 1950), and Zhangixalus omeimontis (Stejneger, 1924).

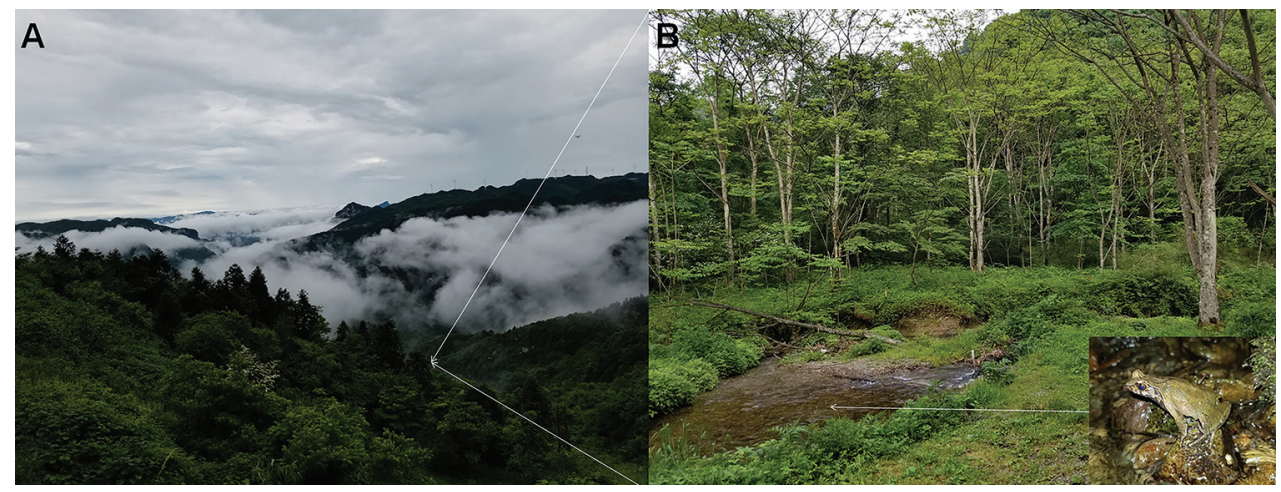

Figure 8. Habitats of Megophrys qianbeiensis sp. nov. in the type locality, Huanglian Nature Reserve, Tongzi County, Guizhou Province, China A landscape of montane forests in the type locality B a mountain stream where toads of the new species live (insert the holotype CIBTZ20190608017standing on the stone). 
Etymology. The specific epithet qianbeiensis refers to northern part of Guizhou, also called "黔”, the province where the type locality of the species belongs to. We propose the common English name "Qianbei horned toad" and Chinese name "Qian Bei Jiao Chan (黔北角蟾)”.

\section{Discussion}

The new species Megophrys qianbeiensis sp. nov. resembles M. spinata and M. sangzhiensis, and detailed comparisons with different data are important for recognizing them. Our molecular phylogenetic data on mitochondrial DNA and morphological comparisons both separated the new species from the two closely related species. Megophrys spinata were reported to be distributed widely through the provinces of Sichuan, Guizhou, Hunan, Chongqing, Yunnan, and Guangxi (Fei et al. 2012), but detailed investigations with multiple data suggested that several populations of the species should contain cryptic species (including Megophrys qianbeiensis sp. nov. and M. sangzhiensis). In recent years, many new species of the genus Megophrys have been gradually described, of which a large part of number was found in China (Frost 2020). To date, among the 106 species of Megophrys, 56 species were discovered in China. Even so, many cryptic species still need to be described just in southern China (Chen et al. 2017; Liu et al. 2018).

South-western China has long been proposed as biodiversity hotspot (Myers et al. 2000). Guizhou Province is an important part of southwest China, especially with the particular environments of karst rocky desertifcation, and knowledge of biodiversity levels and/or patterns are still seriously lacking. Recently, a series of new amphibian species were described from this province (Zhang et al. 2017; Li et al. 2018a, b, 2019a, b; Lyu et al. 2019; Wang et al. 2019c; Luo et al. 2020; Liu et al. 2020; Wei et al. 2020; $\mathrm{Xu}$ et al. 2020), indicating that species diversity of amphibians in this region is highly underestimated. It is urgent for herpetologists to conduct comprehensive and in-depth surveys to explore the level of amphibian species diversity in this region under accelerating climate changes. Obviously, more work should be conducted on detecting the differentiation of the populations and further describe the cryptic species in this region.

\section{Acknowledgements}

We are grateful to the editors and reviewers for their working on the manuscript. This work was supported by National Natural Sciences Foundation of China (NSFC31960099), Key project for bio-diversity conservation, Ministry of Ecology and Environment of People's Republic of China. (Code: 2018-02-06-M2019-49/50), Basic research project of science and technology department of Guizhou Province (Nos. [2020] 1Y083), Science and technology support project of science and technology department of Guizhou Provincial (No. [2020] 4Y029) and Guizhou Provincial Department of Education Youth Science and Technology Talents Growth Project (Nos. KY[2018]455 and KY[2018]468). 


\section{References}

Boulenger GA (1886) Description of a new frog of the genus Megalophrys. Proceedings of the Zoological Society of London1885: 1-850.

Boulenger GA (1887) Description of a new frog of the genus Megalophrys. Annali del Museo Civico di Storia Naturale di Genova Serie 2, 4: 512-513.

Boulenger GA (1889) Description of a new batrachian of the genus Leptobrachium, obtain by M. L. Burma. Annali del Museo Civico di Storia Naturale di Genova. Serie 2, 7: 748-750.

Boulenger GA (1893) Descriptions of three new batrachians from Tonkin. Annals and Magazine of Natural History, Series 7 12: 186-188. https://doi.org/10.1080/00222930308678835

Boulenger GA (1899a) Descriptions of three new reptiles and a new batrachian from Mount Kina Balu, North Borneo. Annals and Magazine of Natural History, Series 7(4):1-453. https://doi.org/10.1080/00222939908678228

Boulenger GA (1899b) On a collection of reptiles and batrachians made by Mr. J. D. La Touche in N.W. Fokien, China. Proceedings of the Zoological Society of London 1899: 159-172.

Boulenger GA (1903) Report on the batrachians and reptiles. In: Annandale N, Robinson HC (Eds) Fasciculi Malayenses. Anthropological and Zoological Results of an Expedition to Perak and the Siamese Malay States 1901-1903 undertaken by Nelson Annandale and Herbert C. Robinson under the auspices of the University of Edinburgh and the University of Liverpool (Vol. 2), Zoology, Part 1: 131-176.

Boulenger GA (1908) A revision of the oriental pelobatid batrachians (genus Megophrys). Proceedings of the Zoological Society of London 78(2): 407-430. https://doi. org/10.1111/j.1096-3642.1908.tb01852.x

Bourret R (1937) Notes herpétologiques sur l'Indochine française. XIV. Les batraciens de la collection du Laboratoire des Sciences Naturelles de l'Université. Descriptions de quinze especes ou variétés nouvelles. Annexe au Bulletin Général de l'Instruction Publique Hanoi, 1937: 5-56.

Che J, Chen HM, Yang JX, Jin JQ, Jiang K, Yuan ZY, Murphy RW, Zhang YP (2012) Universal COI primers for DNA barcoding amphibians. Molecular Ecology Resource 12: 247-258. https://doi.org/10.1111/j.1755-0998.2011.03090.x

Chen JM, Zhou WW, Nikolay A, Poyarkov Jr, Stuart BL, Brown RM, Lathrop A, Wang YY, Yuan ZL, Jiang K, Hou M, Chen HM, Suwannapoom C, Nguyen SN, Duong TV, Papenfuss TJ, Murphy RW, Zhang YP, Che J (2017) A novel multilocus phylogenetic estimation reveals unrecognized diversity in Asia toads, genus Megophrys sensu lato (Anura: Megophryidae). Molecular Phylogenetics and Evolution 106: 28-43. https://doi. org/10.1016/j.ympev.2016.09.004

Delorme M, Dubois A, Grosjean S, Ohler A (2006) Une nouvelle ergotaxinomie des Megophryidae (Amphibia, Anura). Alytes 24: 6-21.

Deuti K, Grosjean S, Nicolas V, Vasudevan K, Ohler A (2017) Nomenclatural puzzle in early Megophrys nomina (Anura, Megophryidae) solved with description of two new species from India (Darjeeling hills and Sikkim). Alytes 34: 20-48.

Dubois A (1987) Miscellanea taxinomica batrachologica (I). Alytes 1987[1986]: 7-95.

Fei L, Hu SQ, Ye CY, Huang YZ (2009) Fauna Sinica. Amphibia (Vol. 2). Anura. Science Press, Beijing, 328-481. [In Chinese] 
Fei L, Ye CY, Huang YZ (1983) Two new subspecies of Megophrys omeimontis Liu from China (Amphibia, Pelobatidae). Acta Herpetologica Sinica. New Series, Chengdu 2(2): 49-52. [In Chinese with English abstract]

Fei L, Ye CY, Huang YZ (1990) Key to Chinese Amphibians. Publishing House for Scientific and Technological, Chongqing, 108-110. [In Chinese]

Fei L, Ye CY, Huang YZ (2001) Colour Handbook Amph. Sichuan, Science Press, Beijing, 138-139. [In Chinese]

Fei L, Ye CY (2005) Two new species of Megophryidae from China. In: Fei et al. (Ed.) The Key and Illustration of Chinese. Sichuan Publishing House of Science and Technology, Chongqing, 253-255. [In Chinese]

Fei L, Ye CY, Jiang JP (2012) Colored atlas of Chinese Amphibians and their distributions. Sichuan Publishing House of Science and Technology, Chengdu, 135-247. [In Chinese]

Fei L, Ye CY (2016) Genus Liuophrys Fei, Ye and Jiang, new genus; Subgenus Atympanophrys (Borealophrys) Fei, Ye and Jiang, new subgenus; Subgenus Atympanophrys (Gigantophrys) Fei, Ye and Jiang, new subgenus; Genus Boulenophrys Fei, Ye and Jiang, 2016, new genus; Subgenus Xenophrys (Tianophrys) Fei, Ye and Jiang, new subgenus. In: Fei L, Ye CY (Eds) Amphibians of China (Vol. (I)). Science Press, Beijing, 611-735.

Frost DR (2020) Amphibian Species of the World: an Online Reference. Version 6.0. Electronic Database. American Museum of Natural History, New York, USA. http://research. amnh.org/herpetology/amphibia/index.html [Accessed on 20 Apr 2020]

Günther ACLG (1864) The reptiles of British India. Ray Society, London, 414-415.

Guindon S, Dufayard JF, Lefort V, Anisimova M, Hordijk W, Gascuel O (2010) New algorithms and methods to estimate maximum-likelihood phylogenies: assessing the performance of PhyML 3.0. Systematic Biology 59(3): 07-321. https://doi.org/10.1093/sysbio/syq010

Hall TA (1999) BIOEDIT: a user-friendly biological sequence alignment editor and analysis program for Windows 95/98/NT. Nucleic Acids Symposium Series 41(41): 95-98.

Hu SX, Zhao EM, Liu CZ (1966) A herpetological survey of the Tsinling and Ta-pa shan region. Acta Zoologica Sinica 18(1): 57-89.

Hu SX, Zhao EM, Liu CZ (1973) A survey of amphibians and reptiles in Kweichow province, including a herpetofauna analysis. Acta Zoologica Sinica 19(2): 149-171.

Huang YZ, Fei L (1981) Two new species of amphibians from Xizang. Acta Zootaxonomica Sinica 6: 211-215.

Inger RF (1989) Four new species of frogs from Borneo. Malayan Nature Journal. Kuala Lumpur 42: 229-243.

Inger RF, Romer JD (1961) A new pelobatid frog of the genus Megophrys from Hong Kong. Fieldiana. Zoology 39(46): 533-538. https://doi.org/10.5962/bhl.title.3373

Inger RF, Stuebing RB, Lian TF (1995) New species and new records of Anurans from Boreno. Raffles Bulletin of Zoology, Singapore 43(1): 115-131.

Inger RF, Iskandar DT (2005) A collection of amphibians from west Sumatra, with description of a new species of Megophrys (Amphibia: Anura). Raffles Bulletin of Zoology, Singapore $133-142$.

Jiang JP, Yuan FR, Xie F, Zheng ZH (2003) Phylogenetic relationships of some species and genera in megophryids inferred from partial sequences of mitochondrial $12 \mathrm{~S}$ and $16 \mathrm{~S}$ rRNA genes. Zoological Research 24: 241-248. [In Chinese with English abstract] 
Jiang JP, Ye CY, Fei L (2008) A New Horn Toad Megophrys sangzhiensis from Hunan, China (Amphibia, Anura). Zoological Research 29(2): 219-222. [In Chinese with English abstract] https://doi.org/10.3724/SP.J.1141.2008.00219

Kuhl H, Van Hasselt JC (1822) Uittreksels uit breieven van de Heeren Kuhl en van Hasselt, aan de Heeren C. J. Temminck, Th. van Swinderen en W. de Haan. Algemeene Konst-en Letter-Bode 7: 99-104.

Lathrop A (1997) Taxonomic review of the megophryid frogs (Anura: Pelobatoidea). Asian Herpetological Research 7: 68-79. https://doi.org/10.5962/bhl.part.18857

Lemmon AR, Moriarty EC (2004) The importance of proper model assumption in Bayesian phylogenetics. Systematic Biology 53(2): 265-277. https://doi. org/10.1080/10635150490423520

Li YL, Jin MJ, Zhao J, Liu ZY, Wang YY, Pang H (2014) Description of two new species of the genus Megophrys (Amphibia: Anura: Megophryidae) from Heishiding Natural Reserve, Fengkai, Guangdong, China, based on molecular and morphological data. Zootaxa 3795(4): 449-471. https://doi.org/10.11646/zootaxa.3795.4.5

Li SZ, Xu N, Lv JC, Jiang JP, Wei G, Wang B (2018a) A new species of the odorous frog genus Odorrana (Amphibia, Anura, Ranidae) from southwestern China. PeerJ 6(e5695): 1-28. https://doi.org/10.7717/peerj.5695

Li SZ, Xu N, Liu J, Jiang JP, Wei G, Wang B (2018b) A New Species of the Asian Toad Genus Megophrys sensu lato (Amphibia: Anura: Megophryidae) from Guizhou Province, China. Asian Herpetological Research 9(4): 224-239. https://doi.org/10.16373/j.cnki.ahr.180072

Li SZ, Wei G, Xu N, Cui JG, Fei L, Jiang JP, Liu J, Wang B (2019a) A new species of the Asian music frog genus Nidirana (Amphibia, Anura, Ranidae) from Southwestern China. PeerJ 7: e7157. https://doi.org/10.7717/peerj.5695

Li SZ, Zhang MH, Xu N, Lv JC, Jiang JP, Liu J, Wei G, Wang B (2019b) A new species of the genus Microhyla (Amphibia: Anura: Microhylidae) from Guizhou Province, China. Zootaxa 4624: 551-575. https://doi.org/10.11646/zootaxa.4624.4.7

Li Y, Zhang DD, Lyu ZT, Wang J, Li YL, Liu ZY, Chen HH, Rao DQ, Jin ZF, Zhang CY, Wang YY (2020) Review of the genus Brachytarsophrys (Anura: Megophryidae), with revalidation of Brachytarsophrys platyparietus and description of a new species from China. Zoological Research 41(2): 105-122. https://doi.org/10.24272/j.issn.2095-8137.2020.033

Liu CZ (1950) Amphibians of Western China. Fieldiana. Zoology Memoires 2: 1-400. https:// doi.org/10.5962/bhl.part.4737

Liu CZ, Hu SQ, Yang HH (1960). Amphibian of Yunnan collected in 1958. Acat Zoological Sinica 12(2): 149-174.

Liu ZY, Zhu TQ, Zeng ZC, Lyu ZT, Wang J, Messenger K, Greenberg A J, Gou ZX, Yang ZH, Shi SH, Wang YY (2018) Prevalence of cryptic species in morphologically uniform taxaFast speciation and evolutionary radiation in Asian frogs. Molecular Phylogenetics and Evolution 127: 723-731. https://doi.org/10.1016/j.ympev.2018.06.020

Liu J, Li SZ, Wei G, Xu N, Cheng YL, Wang B, Wu J (2020) A new species of the Asian toad genus Megophrys sensu lato (Anura: Megophryidae) from Guizhou Province, China. Asian Herpetological Research 11(1): 1-18. https://doi.org/10.16373/j.cnki.ahr.190041 
Lyu ZT, Zeng ZC, Wan H, Yang JH, Li YL, Pang H, Wang YY (2019b) A new species of Amolops (Anura: Ranidae) from China, with taxonomic comments on A. liangshanensis and Chinese populations of $A$. marmoratus. Zootaxa 4609: 247-268. https://doi.org/10.11646/ zootaxa.4609.2.3

Lyu ZT, Li YQ, Zeng ZC, Zhao J, Liu ZL, Guo GX, Wang YY (2020) Four new species of Asian horned toads (Anura, Megophryidae, Megophrys) from southern China. ZooKeys 942: 105-140. https://doi.org/10.3897/zookeys.942.47983

Luo T, Xiao N, Gao K, Zhou J (2020) A new species of Leptobrachella (Anura, Megophryidae) from Guizhou Province, China. ZooKeys 923: 115-140. https://doi.org/10.3897/zookeys.923.47172

Mahony S (2011) Two new species of Megophrys Kuhl \& van Hasselt (Amphibia: Megophryidae), from western Thailand and southern Cambodia. Zootaxa 2734: 23-39. https://doi. org/10.11646/zootaxa.2734.1.2

Mahony S, Sengupta S, Kamei RG, Biju S D (2011) A new low altitude species of Megophrys Kuhl and van Hasselt (Amphibia: Megophryidae), from Assam, Northeast India. Zootaxa 3059: 36-46. https://doi.org/10.11646/zootaxa.3059.1.2

Mahony S, Teeling EC, Biju SD (2013) Three new species of horned frogs, Megophrys (Amphibia: Megophryidae), from northeast India, with a resolution to the identity of Megophrys boettgeri populations reported from the region. Zootaxa 3722(2): 143-169. https://doi. org/10.11646/zootaxa.3722.2.2

Mahony S, Nicole MF, Biju SD, Teeling EC (2017) Evolutionary history of the Asian Horned Frogs (Megophryinae): integrative approaches to time tree dating in the absence of a fossil record. Molecular Phylogenetics and Evolution 34(3): 744-771. https://doi.org/10.1093/ molbev/msw267

Mahony S, Kamei RG, Teeling EC, Biju SD (2018) Cryptic diversity within the Megophrys major species group (Amphibia: Megophryidae) of the Asian Horned Frogs: Phylogenetic perspectives and a taxonomic revision of South Asian taxa, with descriptions of four new species. Zootaxa 4523: 1-96. https://doi.org/10.11646/zootaxa.4523.1.1

Malkmus R, Matsui M (1997) Megophrys kobayashii, ein neuer pelobatider Frosch vom Mount Kinabalu. Sauria, Berlin 19: 31-37.

Mathew R, Sen N (2007) Description of two new species of Megophrys (Amphibia: Anura: Megophryidae) from North-east India. Cobra 1: 18-28.

McGuire JA, Witt CC, Altshuler DL, Remsen JV (2007) Phylogenetic systematics and biogeography of hummingbirds: Bayesian and maximum likelihood analyses of partitioned data and selection of an appropriate partitioning strategy. Systematic Biology 56(5): 837-856. https://doi.org/10.1080/10635150701656360

Messenger KR, Dahn HA, Liang YR, Xie P, Wang Y, Lu CH (2019) A new species of the genus Megophrys Gunther, 1864 (Amphibia: Anura: Megophryidae) from Mount Wuyi, China. Zootaxa 4554(2): 561-583. https://doi.org/10.11646/zootaxa.4554.2.9

Mo XY, Shen YH, Li HH, Wu MS (2010) A new species of Megophrys (Amphibia: Anura: Megophryidae) from the northwestern Hunan Province, China. Current Zoology 56(4): 432-436. https://doi.org/10.1093/czoolo/56.4.432 
Munir M, Hamidy A, Farajallah A, Smith EN (2018) A new Megophrys Kuhl and Van Hasselt (Amphibia: Megophryidae) from southwestern Sumatra, Indonesia. Zootaxa 4442: 389-412. https://doi.org/10.11646/zootaxa.4442.3.3

Munir M, Hamidy A, Matsui M, Iskandar DT, Sidik I, Shimada T (2019) A new species of Megophrys Kuhl \& Van Hasselt (Amphibia: Megophryidae) from Borneo allied to $M$. nasuta. Zootaxa 4679: 1-24. https://doi.org/10.11646/zootaxa.4679.1.1

Myers N, Mittermeier RA, Mittermeier CG, Da Fonseca GAB, Kent J (2000) Biodiversity hotspots for conservation priorities. Nature 403: 853-858. https://doi.org/10.1038/35002501

Nguyen TQ, Pham CT, Nguyen TT, Luong AM, Ziegler T (2020) A new species of Megophrys (Amphibia: Anura: Megophryidae) from Vietnam. Zootaxa 4722: 401-422. https://doi. org/10.11646/zootaxa.4722.5.1

Ohler A, Swan SR, Daltry JC (2002) A recent survey of the amphibian fauna of the Cardamom Mountains, Southwest Cambodia with descriptions of three new species. Raffles Bulletin of Zoology, Singapore 50(2): 465-481.

Ohler A (2003) Revision of the genus Ophryophryne Boulenger, 1903 (Megophryidae) with description of two new species. Alytes 21(1): 23-44.

Pope CH (1929) Four new frogs from Fukien Province, China. American Museum Novitates 352: $1-5$.

Poyarkov NA, Duong Jr TV, Orlov NL, Gogoleva SI, Vassilieva AB, Nguyen LT, Nguyen VDH, Nguyen SN, Che J, Mahony S (2017) Molecular, morphological and acoustic assessment of the genus Ophryophryne (Anura, Megophryidae) from Langbian Plateau, southern Vietnam, with description of a new species. ZooKeys 672: 49-120. https://doi.org/10.3897/ zookeys.672.10624

R Development Core Team (2008) R: a language and environment for statistical computing. Vienna: R Foundation for Statistical Computing. http://www.Rproject.org

Rao DQ, Yang DT (1997) The karyotypes of Megophryinae (Pelobatidae) with a discussion on their classification and phylogenetic relationships. Asian Herpetological Research 7: 93-102. https://doi.org/10.5962/bhl.part.18858

Robert L, Brett C, Simon YWH, Stephane G (2012) PartitionFinder: Combined Selection of Partitioning Schemes and Substitution Models for Phylogenetic Analyses. Molecular Phylogenetics and Evolution 29(6): 1695-1701. https://doi.org/10.1093/molbev/ mss020

Ronquist FR, Huelsenbeck JP (2003) MrBayes3: Bayesian phylogenetic inference under mixed models. Bioinformatics 19(12): 1572-1574. https://doi.org/10.1093/bioinformatics/btg180

Sambrook J, Fritsch EF, Maniatis T (1989) Molecular Cloning: A Laboratory Manual. Cold Spring Harbor Laboratory Press, New York, 1659 pp.

Schlegel H (1858) Handleiding tot de Beoefening der Dierkunde (Vol. 2). Koninklijke Militaire Akademie, Breda, 57 pp. https://doi.org/10.5962/bhl.title.11648

Shen Y H (1994) A new pelobatid toad of the genus Megophrys from China (Anura: Pelobatidae). Zoological Society of China Editor. The $60^{\text {th }}$ Anniversary of the Foundation of the Zoological Society of China, Nanking (China), September 1994. China Science and Technology Publishing House, Nanjing, 603-606.

Simon C, Frati F, Beckenbach A, Crespi B, Liu H, Flook P (1994) Evolution, weighting and phylogenetic utility of mitochondrial gene sequences and a compilation of conserved poly- 
merase chain reaction primers. Annals of the Entomological Society of America 87(6): 651-701. https://doi.org/10.1093/aesa/87.6.651

Sjöander K, Beskow J (2000) Wavesurfer (Anura: Pelobatidae). Acoustical society of China Editor. The International Conference on Spoken Language Processing, Beijing (China), October 2000. Military Yiwen Publishing House, Beijing, 464-467.

Smith MA (1921) New or little-known reptiles and batrachians from southern Annam (IndoChina). Proceedings of the Zoological Society of London 1921: 423-440. https://doi. org/10.1111/j.1096-3642.1921.tb03271.x

Stejneger L (1926) Two new tailless amphibians from western China. Proceedings of the Biological Society of Washington 39: 53-54.

Stuart BL, Chuaynkern Y, Chan-ard T, Inger RF (2006) Three new species of frogs and a new tadpole from eastern Thailand. Fieldiana Zoology, New Series 1543: 1-10. https://doi. org/10.3158/0015-0754(2006)187[1:TNSOFA]2.0.CO;2

Tamura K, Stecher G, Peterson D, Fiipski A, Kumar S (2013). MEGA6: molecular evolutionary genetics analysis, version 6.0. Molecular Biology and Evolution 30: 2725-2729. https://doi.org/10.1093/molbev/mst197

Tapley B, Cutajar T, Mahony S, Nguyen CT, Dau VQ, Nguyen TT, Luong HV, Rowley JJL (2017) The Vietnamese population of Megophrys kuatunensis (Amphibia: Megophryidae) represents a new species of Asian horned frog from Vietnam and southern China. Zootaxa 4344(3): 465-492. https://doi.org/10.11646/zootaxa.4344.3.3

Tapley B, Cutajar TP, Mahony S, Nguyen CT, Dau VQ, Luong AM, Le DT, Nguyen TT, Nguyen TQ, Portway C, Luong HV, Rowley JJL (2018) Two new and potentially highly threatened Megophrys Horned frogs (Amphibia: Megophryidae) from Indochina's highest mountains. Zootaxa 4508: 301-333. https://doi.org/10.11646/zootaxa.4508.3.1

Taylor EH (1920) Philippine Amphibia. Philippine Journal of Science 16: 213-359. https:// doi.org/10.5962/bhl.part.4751

Taylor EH (1962) The amphibian fauna of Thailand. University of Kansas Science Bulletin 43: 265-599. https://doi.org/10.5962/bhl.part.13347

Tian YZ, Sun A (1995) A new species of Megophrys from China (Amphibia: Pelobatidae). Journal of Liupanshui Teachers College 52(3): 11-15. [In Chinese].

Tian WS, Hu QX (1983) Taxonomic study on genus Megophrys, with descriptions of two genera. Acta Herpetologica Sinica 2: 41-48.

Wang J, Liu ZY, Lyu ZT, Wang YY (2017a) A new species of the genus Megophrys (Amphibia: Anura: Megophryidae) from an offshore island in Guangdong Province, southeastern China. Zootaxa 4324(3): 541-556. https://doi.org/10.11646/zootaxa.4324.3.8

Wang YE, Liu BQ, Jiang K, Jin W, Xu JN, Wu CH (2017b) A new species of the Horn Toad of the genus Xenophrys from Zhejiang, China (Amphibia: Megophryidae). Chinese Journal of Zoology 52: 19-29. [in Chinese with English abstract]

Wang YY, Zhang TD, Zhao J, Sung YH, Yang JH, Pang H, Zhang Z (2012) Description of a new species of the genus Megophrys Günther, 1864 (Amphibia: Anura: Megophryidae) from Mount Jinggang, China, based on molecular and morphological data. Zootaxa 3546: 53-67. https://doi.org/10.11646/zootaxa.3546.1.4

Wang YY, Zhao J, Yang JH, Zhou ZM, Chen GL, Liu Y (2014) Morphology, molecular genetics, and bioacoustics support two new sympatric Megophrys (Amphibia: Anura Megophryi- 
dae) species in Southeast China. PLoS ONE 9(4): e93075. https://doi.org/10.1371/journal.pone.0093075

Wang L, Deng XJ, Liu Y, Wu QQ, Liu Z (2019a) A new species of the genus Megophrys (Amphibia: Anura: Megophryidae) from Hunan, China. Zootaxa 4695(4): 301-330. https:// doi.org/10.11646/zootaxa.4695.4.1

Wang J, Lyu ZT, Liu ZY, Liao CK, Zeng ZC, Li YL, Wang YY (2019b) Description of six new species of the subgenus Panophrys within the genus Megophrys (Anura, Megophryidae) from southeastern China based on molecular and morphological data. ZooKeys 851: 113-164. https://doi.org/10.3897/zookeys.851.29107

Wang J, Li YL, Li Y, Chen H-H, Zeng YJ, Shen JM, Wang Y-Y (2019c) Morphology, molecular genetics, and acoustics reveal two new species of the genus Leptobrachella from northwestern Guizhou Province, China (Anura, Megophryidae). ZooKeys 848: 119-154. https:// doi.org/10.3897/zookeys.848.29181

Wang B, Wu YQ, Peng JW, Shi SC, Lu NN, Wu J (2020) A new Megophrys Kuhl and Van Hasselt (Amphibia: Megophryidae) from southeastern China. ZooKeys 851: 113-164. https:// doi.org/10.3897/zookeys.904.47354

Wei G, Li SZ, Liu J, Cheng YL, Xu N, Wang B (2020) A new species of the Music frog Nidirana (Anura, Ranidae) from Guizhou Province, China. ZooKeys 904: 63-87. https:// doi.org/10.3897/zookeys.904.39161

Wu YH, Suwannapoom C, Jr. Poyarkov NA, Chen JM, Pawangkhanant P, Xu K, Jin JQ, Murphy RW, Che J (2019) A new species of the genus Xenophrys (Anura: Megophryidae) from northern Thailand. Zoological Research 40: 564-574. https://doi.org/10.24272/j. issn.2095-8137.2019.032

Xu N, Li SZ, Liu J, Wei G, Wang B (2020) A new species of the horned toad Megophrys Kuhl \& Van Hasselt, 1822 (Anura, Megophryidae) from southwest China. ZooKeys 943: 119-144. https://doi.org/10.3897/zookeys.943.50343

Yang JH, Wang J, Wang YY (2018) A new species of the genus Megophrys (Anura: Megophryidae) from Yunnan Province, China. Zootaxa 4413: 325-338. https://doi.org/10.11646/ zootaxa.4413.2.5

Ye CY, Fei L (1992) A new Pelobatid toda of the genus Megophrys from Xizang, China. Acta Herpetologica Sinica 1-2: 50-52. [In Chinese]

Ye CY, Fei L (1995) Taxonomic studies on the small type Megophrys in China including descriptions of the new species (subspecies) (Pelobatidae: genus Megophrys). Herpetologica Sinica 4-5: 72-81. [In Chinese]

Ye CY, Fei L, Xie F (2007) A new species of Megophryidae Megophrys baolongensis from China (Amphibia, Anura). Herpetologica Sinica 11:38-41. [In Chinese]

Zhang Y, Li G, Xiao N, Li J, Pan T, Wang H, Zhang B, Zhou J (2017) A new species of the genus Xenophrys (Amphibia: Anura: Megophryicae) from Libo County, Guizhou, China. Asian Herpetological Research 8: 75-85. https://doi.org/10.16373/j.cnki.ahr.160041

Zhao J, Yang JH, Chen GL, Chen CQ, Wang YY (2014) Description of a new species of the genus Brachytarsophrys Tian and Hu, 1983 (Amphibia: Anura: Megophryidae) from Southern China based on molecular and morphological data. Asian Herpetological Research 5(3): 150-160. https://doi.org/10.3724/SP.J.1245.2014.00150 


\section{Supplementary material I}

Table S1. Uncorrected p-distances between the Megophrys species based on COI gene sequences

Authors: Haijun Su, Shengchao Shi, Yanqing Wu, Guangrong Li, Xiaogang Yao, Bin Wang, Shize Li

Data type: molecular data

Copyright notice: This dataset is made available under the Open Database License (http://opendatacommons.org/licenses/odbl/1.0/). The Open Database License $(\mathrm{ODbL})$ is a license agreement intended to allow users to freely share, modify, and use this Dataset while maintaining this same freedom for others, provided that the original source and author(s) are credited.

Link: https://doi.org/10.3897/zookeys.974.56070.suppl1

\section{Supplementary material 2}

Table S2. Diagnostic characters separating the new species described in this study from other species of Megophrys

Authors: Bin Wang

Data type: morphological data

Copyright notice: This dataset is made available under the Open Database License (http://opendatacommons.org/licenses/odbl/1.0/). The Open Database License $(\mathrm{ODbL})$ is a license agreement intended to allow users to freely share, modify, and use this Dataset while maintaining this same freedom for others, provided that the original source and author(s) are credited.

Link: https://doi.org/10.3897/zookeys.974.56070.suppl2 